\title{
Adaptability of Bt and NBt Cotton Hybrids in Different Locations
} in Central and Southern India

\author{
B.H. Nagappa ${ }^{*}$ and B.M. Khadi \\ Agricultural Research Station (UAS, Dharwad), Hanumanamatti, Ranebennur Taluk, \\ Haveri District, India \\ *Corresponding author
}

A B S T R A C T

\begin{tabular}{|l|}
\hline Ke y w or d s \\
Stability, Cotton, \\
Adaptability, $\mathrm{G} \times \mathrm{E}$ \\
interaction
\end{tabular}

\section{Introduction}

Cotton, white gold rules the world of textile moreover it is unchallenged as a natural textile fibre, despite the advent of a multitude of other fibres. It is an important fibre and food crop of nearly 100 countries with China, India, United States, Pakistan and Brazil being five of the largest producers of cotton. Since the discovery of the Mohen-jodaro relics the history of cotton and cotton manufacture came to be treated as beginning from times of the ancient Indus valley civilization, which flourished in India about 5000 years ago.

Gossypium hirsutum, G. barbadense, G. arboreum and $G$. herbaceum are grown in varied agro-climate in northern, central and southern India from $9^{0} \mathrm{~N}$ latitude to $31^{0} \mathrm{~N}$ latitude. G. hirsutum is the principal cultivated cotton and accounts for about 90 per cent of the world cotton production. G. barbadense accounts for about eight per cent of the world cotton production. The estimated area under cotton during 2008 to 2009 in India was 93.73 lakh ha with production and productivity of 290 lakh bales and $526 \mathrm{~kg}$ lint per ha, respectively (Sharma et al., 2009). In the year of 2008 in Karnataka, cotton was grown over an area of 3.5 lakh ha with 8.9 lakh bales production. India has 20 per cent of total cotton growing area of the world and contributes over 12 per cent to total production. 
In India, cotton is cultivated in diverse situations ranging from irrigated situation characterized by intensive management to assured rainfed situation where moisture is not a constraint to dry rainfed situation characterized by low rainfall (less than 500 $\mathrm{mm}$ ) accompanied by high temperature and high intensity.

To achieve the target of increasing cotton production at national level, it is essential that productivity of cotton increases in all these situations. In this regard the adaptability of genotypes in different environment is very important. Thereby the present investigation to identify Bt and non Bt hybrids in South and Central India was taken up.

\section{Materials and Methods}

To analyze the data over three environments the stability model proposed by Eberhart and Russell (1966) was adopted. This model involves the estimation of three parameters which are defined by a mathematical formula.

$\mathrm{Y}_{\mathrm{ij}}=\mu_{\mathrm{i}}+\beta_{\mathrm{i}} \mathrm{I}_{\mathrm{j}}+\mathrm{S}_{\mathrm{ij}}$

Where, $Y_{i j}=$ Mean of the $i^{\text {th }}$ variety at the $j^{\text {th }}$ environment, $\mu_{i}=$ Mean of $i^{\text {th }}$ variety over all environments, $\beta_{i}=$ The regression coefficient that measures the response of $i^{\text {th }}$ variety to varying environments, $S_{i j}=$ The deviation from regression of the $i^{\text {th }}$ variety at the $j^{\text {th }}$ environment and $\mathrm{I}_{\mathrm{j}}=$ The environmental index obtained by subtracting the grand mean from the mean of all varieties at the $j^{\text {th }}$ environment.

These parameters were calculated as follows

Environmental index

$I_{j}=\frac{\Sigma_{j} Y_{i j}}{t}-\frac{\sum_{i} \Sigma_{j} Y_{i j}}{\text { t. } s}$

Where, $\mathrm{t}=$ Number of varieties, $\mathrm{s}=$ Number of environments, with $\Sigma_{\mathrm{j}} \mathrm{I}_{\mathrm{j}}=0$

The regression coefficient for each variety (bi)

$b_{i}=\frac{\Sigma_{j} Y_{i j} I_{j}}{\Sigma_{j} I^{2}}$

Deviation from regression

$S^{2} d_{i}=\frac{\sum_{j} \delta_{i j}^{2}}{S-2}-\frac{S_{e}^{2}}{r}$

Where, $S^{2} e / r=$ Mean square for (estimate of) pooled error

Predicted performance of the genotypes

$\mathrm{Y}_{\mathrm{ij}}=\overline{\mathrm{X}}_{\mathrm{i}}+\mathrm{b}_{\mathrm{i}} \mathrm{I}_{\mathrm{j}}$

Where, $X_{\mathrm{i}}$ is the estimate of $\mu$,

In this model the variance due to environments and genotype $\times$ environment are partitioned into environment (linear), genotype $\times$ environment (linear) and deviation from the regression coefficient.

\section{Genotype-environment interaction}

The magnitude of GE interaction was assessed for each character and each genotype. This was worked out as per the procedure suggested by Eberhart and Russell (1966).

The analysis of variance for stability as per this model is algebraically represented as below.

Where, $\mathrm{n}=$ number of environments, $\mathrm{r}=$ number of replications, $\mathrm{g}=$ number of genotypes, $\mathrm{CF}=$ Correction factor.

To test the significance of pooled deviation mean square against the pooled error mean square. 
$\mathrm{F}=\frac{\mathrm{MS}_{3}}{\mathrm{MS}_{4}}$

If pooled deviation mean square is found significant then it would be the appropriate denominator to test the significance of all components including genotypes, genotype $x$ environment (linear). Otherwise pooled error mean square is appropriate denominator.

To test the significance of the differences among the means of genotypes.

$$
\mathrm{F}=\frac{\mathrm{MS}_{1}}{\mathrm{MS}_{3}}
$$

To test that the genotypes do not differ for their regression on environmental index

$\mathrm{F}=\frac{\mathrm{MS}_{2}}{\mathrm{MS}_{3}}$

Further, ' $t$ ' test is used to test the significance of deviation of ' $b_{i}$ ' from unity

$$
\mathrm{t}=\frac{\left|1-\mathrm{b}_{\mathrm{i}}\right|}{\mathrm{S.E}\left(\mathrm{b}_{\mathrm{i}}\right)}
$$

Where,

$$
\mathrm{SE}(\mathrm{bi})=\frac{\overline{\Sigma \mathrm{j} \delta 2 \mathrm{ij} / \mathrm{n}-2}}{\Sigma \mathrm{j} \mathrm{I} 2 \mathrm{j}}
$$

Where, $\delta_{\mathrm{ij}}^{2}$ is the deviation of $\mathrm{i}^{\text {th }}$ variety in $\mathrm{j}^{\text {th }}$ environment from regression

To test individual deviation from linear regression

$\mathrm{F}=$ Pooled error mean square $\left(\frac{\Sigma_{\mathrm{j}} \delta_{\mathrm{ij}}}{(\mathrm{n}-2)}\right) /$

A joint consideration of three parameters, that is
The mean performance of the genotype over environments (location), $\mathrm{Xi}$

Regression coefficient $b_{i}$ and

The deviation from linear regression $\mathrm{S}^{2} \mathrm{~d}_{\mathrm{i}}$, are used to define stability of genotype (variety).

The estimate of deviation from regression suggests the degree of reliance that should be put to linear regression in interpretation of data. If these values are significantly deviating from zero, the expected phenotype cannot be predicted satisfactorily. When deviations are non-significant, the conclusions, may be drawn by joint consideration of mean yield and regression values (Finlay and Wilkinson, 1963 and Eberhart and Russell, 1966) as below.

Regression value of unity is interpreted as average stability since the average slope over all varieties on the environment index will be unity.

\section{Results and Discussion}

The detailed ANOVA for stability (Table 13) revealed significant differences among the genotypes with respect to yield, twenty boll weight, ginning outturn, seed index, oil content at 1 per cent level and monopodial branches at 5 per cent level. But, there was no significant difference in sympodia, bolls per plant, fuzz per cent, hull per cent and kernel per cent. Variance due to environment was significant for all traits at 1 per cent level. $G \times$ E interaction was found significant for yield, plant height, bolls per plant, oil percentage, fuzz per cent at 1 per cent level and monopodia and seed index showed significance at 5 per cent level. Remaining characters viz., sympodia, 20 boll weight, hull per cent and kernel per cent and ginning outturn (\%) recorded non-significant $\mathrm{G} \times \mathrm{E}$ interaction. $\mathrm{G} \times \mathrm{E}$ (linear) was found 
significant for sympodial branches, bolls per plant, ginning outturn per cent, fuzz per cent, hull per cent and kernel per cent at 1 per cent level. Besides, significant pooled deviations show that performance of genotypes fluctuated from the linear path of response to environment for all characters except ginning outturn per cent.

Genetic adaptability studies through stability parameters (mean performance, bi and $S^{2}$ di) for different traits has been presented in Table 29.

\section{Stability analysis}

Genotype $\times$ environmental interactions are of major importance to plant breeders in developing improved varieties. These interactions are usually present in any biological experiment, reducing the progress from selections (Comstock and Moll, 1963). Selection of genotypes that interact less with the environments in which they are grown is known to reduce genotype $\times$ environment interactions (Allard and Bradshaw, 1964) and aids the breeder in developing stable genotypes.

The present study comprising $24 \mathrm{Bt}$ hybrids and their non-Bt counterpart hybrids were evaluated across three locations viz., Dharwad, Bagalkot and Nagpur for 12 quantitative traits to know the prevalence of $\mathrm{G} \times \mathrm{E}$ interactions and stability of performance of hybrids across locations for the characters presented in Table $1,2,3,4,5,6,7,8,9,10,11$ and12. The results revealed significant differences among the hybrids for different traits studied in each of the environments and after testing for homogeneity of errors across environments, the data was considered for pooling, which is a prerequisite for two way analysis.

In the two-way analysis of variance (Table pooled analysis) presented in Table 14. The mean square due to genotype $\times$ environmental interactions was found significant for seed cotton yield ( $\mathrm{kg} / \mathrm{ha})$, plant height, number of bolls per plant, oil per cent, fuzz content, monopodial branches and ginning outturn which exhibited significance at 1 and 5 per cent suggesting differential response of the genotypes to varying environments and mean squares due to environment was also observed to be significant for all the traits justifying the selection of sites for the present investigation. A review of past work indicated considerable $\mathrm{G} \times \mathrm{E}$ interaction operating in cotton (Bhatade and Bhale, 1983; Dani, 1999; Blanche et al., 2006; Reddy and Reddy, 2007; El-Kadi et al., 2007; Vannirajan, 2007 and Saini et al., 2008).

Further, the analysis of variance for stability (Eberhart and Russell, 1964) also revealed significant differences among the hybrids for seed cotton yield (kg/ha), 20-boll weight, ginning outturn, seed index, oil content and monopodial branches. The additive environmental variance was found to be of considerable magnitude as indicated by the significance of variance due to environment (linear) for all the characters except kernel content. The mean squares due to pooled deviation was found significant for all the characters except seed cotton yield (kg/ha), thus implying that there is no simple relationship existing between genotypes and the environments and therefore, it is rather difficult to predict the performance of the genotypes across environments.

Bhatade and Bhale (1983), Deshpande and Bhale (1983), Blanche et al., (2006), Elkadi et al., (2007), Saini et al.,(2008), Reddy and Reddy (2007), for oil content, obtained significance for genotype $\times$ environment (linear). But, found significance for pooled deviation which indicated that part of variation in the performance of genotypes is predictable (Eberhart and Russell, 1964). 
The more pronounced linearity for the character oil content could largely be explained by differences in regression slopes. This obviously means that fairly accurate prediction of the genotypic performance of genotypes is possible for oil content.

The mean performance of genotypes in the present study varied with the environment in response to change in environments. As the genotypes did not respond uniformly, the mean performance of genotypes i.e., population mean or site mean (Eberhart and Russell, 1964) varied across environments. A single parameter based on site mean, the "Environment index" was introduced which is the deviation of site mean from overall grand mean. Once the significant genotype $x$ environment interaction is observed for a given trait, the next task would be to identify the stable genotype, which interacts moderately with the environments giving a near consistent performance across environments.

The regression coefficient and deviation from regression of each variety presented in Table 15.

Finlay and Wilkinson (1963) suggested that a genotype is considered as stable, if it had high mean and unit regression coefficient (bi $=1$ ). However, the model is based only on linear regression which could simply be regarded as a measure of response of a particular genotype. Eberhart and Russell (1964) suggested both linear and non-linear $\left(\mathrm{S}^{2} \mathrm{~d}_{\mathrm{i}}\right)$ components of genotype $\times$ environmental interactions.

According to this model, the genotype is considered as ideal and stable when it possesses high or low mean (depending on the character) with regression coefficient around unity $(\mathrm{bi}=1)$ and deviation from regression coefficient $\left(S^{2} d_{i}\right)$ being non-significant.
A stable genotype is one with a regression slope near to unity, insignificant deviation from regression line and having higher mean value for the trait.

Patel et al., (1999) observed 64 Gossypium hirsutum hybrids and their 20 parents for yield at three locations. Hybrids from four crosses possessed average stability and gave high yield. All parents gave lower yields, but showed greater stability than the hybrids. Similar results were observed by (Eberhart and Russell, 1964), Deshpande and Bhale (1983), Bhatade and Bhale (1983) and Ansingkar and Bhale (1984), El-Okkia et al., (1982), El-Dayim et al., (1982), Rao et al., (1989), Bhatade et al., (1995), Basu (1996), Baloch et al., (1994), Opondo and Ombakho (1997), Wondima (2000), Stoilova and Dechev (2002), El-Feki et al., (2002), Anandan et al., (2003), Badra (2003), Nanjundan et al., (2004), Nirania (2004), Anandan et al., (2005), Pund and Dev (2006), Xing Chao et al., (2007) and Saini et al., (2008).

So far many Bt hybrids have been released and identification of location specific and well adapted Bt hybrids is the pressing need. Thereby this investigation was planned and executed. The results are presented in Table 16.

K-5038 Bt (1820.33 kg/ha), NCEN-2R NBt (1763.33 kg/ha), JKCH-2245 NBt (1665.33 kg/ha), KDCHH-441 Bt (1651 kg/ha), Dhruva Bt (1643 kg/ha) and JKCH-99 Bt (1577.33 $\mathrm{kg} / \mathrm{ha}$ ) hybrids were found suitable for cultivation at all the three locations indicating their wider adaptability based on stability parameter. On an average, seed cotton yield of all these hybrids was significantly higher than check. The deviation from regression coefficient was also insignificant for these hybrids and regression coefficient of all these hybrids did not significantly deviate from 1 . 
Table.1 Mean performance of Bt and non-Bt hybrids across three environments for oil content (\%)

\begin{tabular}{|c|c|c|c|c|c|c|c|c|c|c|c|c|c|c|c|c|c|c|c|c|}
\hline \multirow[t]{2}{*}{ Genotypes } & \multicolumn{5}{|c|}{$E_{1}$ : Nagpur } & \multicolumn{5}{|c|}{$\mathrm{E}_{2}$ : Dharwad } & \multicolumn{5}{|c|}{ E3: Bagalkot } & \multirow{2}{*}{$\begin{array}{c}\text { Mean } \\
\text { Bt }\end{array}$} & \multirow{2}{*}{$\begin{array}{l}\text { Mean } \\
\text { NBt }\end{array}$} & \multicolumn{3}{|c|}{ Across locations } \\
\hline & Bt & NBt & $\begin{array}{c}\text { \% over } \\
\text { NBt }\end{array}$ & $\begin{array}{c}\% \\
\text { over } \\
\text { check }\end{array}$ & $\begin{array}{c}\text { \% over } \\
\text { population } \\
\text { mean }\end{array}$ & Bt & NBt & $\begin{array}{c}\text { \% over } \\
\text { NBt }\end{array}$ & $\begin{array}{c}\% \\
\text { over } \\
\text { check }\end{array}$ & $\begin{array}{c}\% \text { over } \\
\text { population } \\
\text { mean }\end{array}$ & Bt & NBt & $\begin{array}{c}\text { \% over } \\
\text { NBt }\end{array}$ & $\begin{array}{c}\% \\
\text { over } \\
\text { check }\end{array}$ & $\begin{array}{c}\% \text { over } \\
\text { population } \\
\text { mean }\end{array}$ & & & $\begin{array}{c}\text { \% over } \\
\text { NBt }\end{array}$ & $\begin{array}{c}\% \\
\text { over } \\
\text { check }\end{array}$ & $\begin{array}{c}\% \\
\text { over } \\
\text { popu } \\
\text { lation } \\
\text { mean }\end{array}$ \\
\hline JKCH-2245 & 20.43 & 21.75 & -6.07 & 5.85 & 12.69 & 19.20 & 18.10 & 6.08 & 8.17 & 1.97 & 20.35 & 20.70 & -1.69 & -1.93 & -0.24 & 19.99 & 20.18 & -0.94 & 3.74 & 4.72 \\
\hline K-5038 & 21.73 & 21.31 & 1.97 & 12.59 & 10.41 & 19.25 & 16.65 & 15.62 & 8.45 & -6.20 & 21.55 & 18.75 & 14.93 & 3.86 & -9.64 & 20.84 & 18.90 & 10.26 & 8.15 & -1.92 \\
\hline KDCHH-441 & 20.67 & 18.71 & 10.48 & 7.10 & -3.06 & 18.25 & 17.10 & 6.73 & 2.82 & -3.66 & 19.45 & 17.75 & 9.58 & -6.27 & -14.46 & 19.46 & 17.85 & 9.02 & 0.99 & -7.37 \\
\hline JK-Indra & 20.83 & 21.20 & -1.75 & 7.93 & 9.84 & 18.85 & 18.65 & 1.07 & 6.20 & 5.07 & 21.40 & 21.75 & -1.61 & 3.13 & 4.82 & 20.36 & 20.53 & -0.83 & 5.66 & 6.54 \\
\hline RCH-2 & 19.30 & 19.71 & -2.08 & 0.00 & 2.12 & 17.75 & 17.10 & 3.80 & 0.00 & -3.66 & 20.75 & 17.60 & 17.90 & 0.00 & -15.18 & 19.27 & 18.14 & 6.23 & 0.00 & -5.86 \\
\hline JK-Ishwar & 21.74 & 20.45 & 6.31 & 12.64 & 5.96 & 18.00 & 17.15 & 4.96 & 1.41 & -3.38 & 21.50 & 21.25 & 1.18 & 3.61 & 2.41 & 20.41 & 19.62 & 4.03 & 5.92 & 1.82 \\
\hline JКСН-1947 & 15.16 & 18.56 & -18.32 & -21.45 & -3.83 & 15.80 & 17.10 & -7.60 & -10.99 & -3.66 & 14.60 & 18.00 & -18.89 & -29.64 & -13.25 & 15.19 & 17.89 & -15.09 & -21.17 & -7.16 \\
\hline JKCH-22 & 15.01 & 19.77 & -24.08 & -22.23 & 2.44 & 16.85 & 19.15 & -12.01 & -5.07 & 7.89 & 13.95 & 16.75 & -16.72 & -32.77 & -19.28 & 15.27 & 18.56 & -17.73 & -20.76 & -3.68 \\
\hline Ankur-651 & 18.46 & 18.94 & -2.53 & -4.35 & -1.87 & 17.95 & 17.85 & 0.56 & 1.13 & 0.56 & 17.00 & 17.12 & -0.70 & -18.07 & -17.49 & 17.80 & 17.97 & -0.95 & -7.63 & -6.75 \\
\hline JKCH-1050 & 17.46 & 12.40 & 40.81 & -9.53 & -35.75 & 17.00 & 17.45 & -2.58 & -4.23 & -1.69 & 10.90 & 14.05 & -22.42 & -47.47 & -32.29 & 15.12 & 14.63 & 3.35 & -21.54 & -24.08 \\
\hline PCH-2270 & 18.12 & 20.76 & -12.72 & -6.11 & 7.56 & 17.50 & 16.75 & 4.48 & -1.41 & -5.63 & 18.35 & 21.15 & -13.24 & -11.57 & 1.93 & 17.99 & 19.55 & -7.98 & -6.64 & 1.45 \\
\hline NCEN-3R & 20.81 & 20.83 & -0.10 & 7.82 & 7.93 & 16.62 & 16.40 & 1.34 & -6.37 & -7.61 & 20.40 & 21.30 & -4.23 & -1.69 & 2.65 & 19.28 & 19.51 & -1.18 & 0.05 & 1.25 \\
\hline КDCHН-9810 & 20.36 & 19.61 & 3.82 & 5.49 & 1.61 & 18.25 & 17.00 & 7.35 & 2.82 & -4.23 & 21.55 & 19.60 & 9.95 & 3.86 & -5.54 & 20.05 & 18.74 & 6.99 & 4.05 & -2.75 \\
\hline NCEN-2R & 20.35 & 20.72 & -1.79 & 5.44 & 7.36 & 17.25 & 17.35 & -0.58 & -2.82 & -2.25 & 21.02 & 19.75 & 6.43 & 1.30 & -4.82 & 19.54 & 19.27 & 1.40 & 1.40 & 0.00 \\
\hline JKCH-266 & 20.10 & 14.52 & 38.43 & 4.15 & -24.77 & 18.45 & 15.00 & 23.00 & 3.94 & -15.49 & 14.50 & 19.20 & -24.48 & -30.12 & -7.47 & 17.68 & 16.24 & 8.87 & -8.25 & -15.72 \\
\hline Dhruva & 21.94 & 22.35 & -1.83 & 13.68 & 15.80 & 17.95 & 17.00 & 5.59 & 1.13 & -4.23 & 22.75 & 21.55 & 5.57 & 9.64 & 3.86 & 20.88 & 20.30 & 2.86 & 8.35 & 5.35 \\
\hline K-5316 & 21.34 & 22.50 & -5.16 & 10.57 & 16.58 & 18.00 & 18.00 & 0.00 & 1.41 & 1.41 & 20.55 & 22.20 & -7.43 & -0.96 & 6.99 & 19.96 & 20.90 & -4.50 & 3.58 & 8.46 \\
\hline KDCHH-9632 & 21.40 & 20.90 & 2.39 & 10.88 & 8.29 & 17.10 & 16.60 & 3.01 & -3.66 & -6.48 & 20.70 & 20.25 & 2.22 & -0.24 & -2.41 & 19.73 & 19.25 & 2.49 & 2.39 & -0.10 \\
\hline JK-Varun & 15.75 & 18.92 & -16.75 & -18.39 & -1.97 & 14.73 & 17.50 & -15.83 & -17.01 & -1.41 & 15.50 & 16.45 & -5.78 & -25.30 & -20.72 & 15.33 & 17.62 & -13.00 & -20.45 & -8.56 \\
\hline PCH-2171 & 17.51 & 19.46 & -10.02 & -9.27 & 0.83 & 17.55 & 17.65 & -0.57 & -1.13 & -0.56 & 16.20 & 19.80 & -18.18 & -21.93 & -4.58 & 17.09 & 18.97 & -9.91 & -11.31 & -1.56 \\
\hline JK-Durga & 16.78 & 14.56 & 15.25 & -13.06 & -24.56 & 15.00 & 16.00 & -6.25 & -15.49 & -9.86 & 16.55 & 16.75 & -1.19 & -20.24 & -19.28 & 16.11 & 15.77 & 2.16 & -16.40 & -18.16 \\
\hline JKCH-99 & 20.60 & 19.75 & 4.30 & 6.74 & 2.33 & 17.95 & 17.85 & 0.56 & 1.13 & 0.56 & 20.50 & 18.25 & 12.33 & -1.20 & -12.05 & 19.68 & 18.62 & 5.69 & 2.13 & -3.37 \\
\hline JKCH-1945 & 17.81 & 19.16 & -7.05 & -7.72 & -0.73 & 16.10 & 16.70 & -3.59 & -9.30 & -5.92 & 13.75 & 19.20 & -28.39 & -33.73 & -7.47 & 15.89 & 18.35 & -13.41 & -17.54 & -4.77 \\
\hline JK-Gowri & 21.25 & 21.31 & -0.28 & 10.10 & 10.41 & 17.00 & 17.80 & -4.49 & -4.23 & 0.28 & 19.75 & 20.15 & -1.99 & -4.82 & -2.89 & 19.33 & 19.75 & -2.13 & 0.31 & 2.49 \\
\hline Mean & 19.37 & 19.51 & & & & 17.43 & 17.25 & & & & 18.48 & 19.14 & & & & 18.43 & 18.63 & & & \\
\hline SEm \pm & \multicolumn{2}{|c|}{0.86} & & & & \multicolumn{2}{|c|}{0.64} & & & & \multicolumn{2}{|c|}{0.68} & & & & \multicolumn{2}{|c|}{0.30} & & & \\
\hline CD at 5\% & \multicolumn{2}{|c|}{2.47} & & & & \multicolumn{2}{|c|}{1.82} & & & & \multirow{2}{*}{\multicolumn{2}{|c|}{$\begin{array}{l}1.94 \\
2.59\end{array}$}} & & & & \multicolumn{2}{|c|}{0.83} & & & \\
\hline CD at $1 \%$ & \multicolumn{2}{|c|}{3.29} & & & & \multicolumn{2}{|c|}{2.43} & & & & & & & & & \multicolumn{2}{|c|}{1.09} & & & \\
\hline
\end{tabular}


Table.2 Mean performance of Bt and non-Bt hybrids across three environments for kernel content (\%)

\begin{tabular}{|c|c|c|c|c|c|c|c|c|c|c|c|c|c|c|c|c|c|c|c|c|}
\hline \multirow[t]{2}{*}{ Genotypes } & \multicolumn{5}{|c|}{$E_{1}$ : Nagpur } & \multicolumn{5}{|c|}{$E_{2}$ : Dharwad } & \multicolumn{5}{|c|}{$E_{3}$ : Bagalkot } & \multirow{2}{*}{$\begin{array}{c}\text { Mean } \\
\text { Bt }\end{array}$} & \multirow{2}{*}{$\begin{array}{l}\text { Mean } \\
\text { NBt }\end{array}$} & \multicolumn{3}{|c|}{ Across locations } \\
\hline & Bt & NBt & $\begin{array}{c}\text { \% over } \\
\text { NBt }\end{array}$ & $\begin{array}{c}\begin{array}{c}\% \\
\text { over } \\
\text { check }\end{array} \\
\end{array}$ & $\begin{array}{c}\% \text { over } \\
\text { population } \\
\text { mean }\end{array}$ & Bt & NBt & $\begin{array}{c}\text { \% over } \\
\text { NBt }\end{array}$ & $\begin{array}{c}\% \\
\text { over } \\
\text { check }\end{array}$ & $\begin{array}{c}\text { \% over } \\
\text { population } \\
\text { mean }\end{array}$ & Bt & NBt & $\begin{array}{c}\text { \% over } \\
\text { NBt }\end{array}$ & $\begin{array}{c}\begin{array}{c}\% \\
\text { over } \\
\text { check }\end{array} \\
\end{array}$ & $\begin{array}{c}\% \text { over } \\
\text { population } \\
\text { mean }\end{array}$ & & & $\begin{array}{c}\text { \% over } \\
\text { NBt }\end{array}$ & $\begin{array}{c}\begin{array}{c}\% \\
\text { over } \\
\text { check }\end{array} \\
\end{array}$ & $\begin{array}{c}\% \\
\text { over } \\
\text { popu } \\
\text { lation } \\
\text { mean }\end{array}$ \\
\hline JKCH-2245 & 63.93 & 63.95 & -0.03 & -11.58 & -11.55 & 62.80 & 63.70 & -1.41 & 1.45 & 2.91 & 54.20 & 54.15 & 0.09 & -7.43 & -7.51 & 60.31 & 60.60 & -0.48 & -6.13 & -5.68 \\
\hline K-5038 & 61.10 & 64.30 & -4.98 & -15.49 & -11.07 & 62.05 & 62.05 & 0.00 & 0.24 & 0.24 & 63.45 & 56.95 & 11.41 & 8.37 & -2.73 & 62.20 & 61.10 & 1.80 & -3.19 & -4.90 \\
\hline KDCHH-441 & 69.70 & 64.15 & 8.65 & -3.60 & -11.27 & 62.60 & 64.55 & -3.02 & 1.13 & 4.28 & 56.10 & 52.45 & 6.96 & -4.18 & -10.42 & 62.80 & 60.38 & 4.01 & -2.26 & -6.02 \\
\hline JK-Indra & 84.35 & 60.95 & 38.39 & 16.67 & -15.70 & 55.30 & 59.70 & -7.37 & -10.66 & -3.55 & 60.15 & 53.95 & 11.49 & 2.73 & -7.86 & 66.60 & 58.20 & 14.43 & 3.66 & -9.42 \\
\hline RCH-2 & 72.30 & 66.60 & 8.56 & 0.00 & -7.88 & 61.90 & 63.90 & -3.13 & 0.00 & 3.23 & 58.55 & 59.00 & -0.76 & 0.00 & 0.77 & 64.25 & 63.17 & 1.71 & 0.00 & -1.68 \\
\hline JK-Ishwar & 73.60 & 71.00 & 3.66 & 1.80 & -1.80 & 66.70 & 61.45 & 8.54 & 7.75 & -0.73 & 55.60 & 48.20 & 15.35 & -5.04 & -17.68 & 65.30 & 60.22 & 8.44 & 1.63 & -6.27 \\
\hline JKCH-1947 & 50.90 & 67.35 & -24.42 & -29.60 & -6.85 & 66.25 & 64.15 & 3.27 & 7.03 & 3.63 & 57.85 & 56.05 & 3.21 & -1.20 & -4.27 & 58.33 & 62.52 & -6.70 & -9.21 & -2.69 \\
\hline JKCH-22 & 71.90 & 68.30 & 5.27 & -0.55 & -5.53 & 60.40 & 66.55 & -9.24 & -2.42 & 7.51 & 56.55 & 57.35 & -1.39 & -3.42 & -2.05 & 62.95 & 64.07 & -1.75 & -2.02 & -0.28 \\
\hline Ankur-651 & 56.50 & 58.25 & -3.00 & -21.85 & -19.43 & 66.15 & 56.20 & 17.70 & 6.87 & -9.21 & 62.10 & 51.90 & 19.65 & 6.06 & -11.36 & 61.58 & 55.45 & 11.06 & -4.16 & -13.70 \\
\hline JKCH-1050 & 66.10 & 63.55 & 4.01 & -8.58 & -12.10 & 62.75 & 63.60 & -1.34 & 1.37 & 2.75 & 55.40 & 55.95 & -0.98 & -5.38 & -4.44 & 61.42 & 61.03 & 0.64 & -4.40 & -5.01 \\
\hline 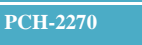 & 67.35 & 65.15 & 3.38 & -6.85 & -9.89 & 63.25 & 57.55 & 9.90 & 2.18 & -7.03 & 59.60 & 55.60 & 7.19 & 1.79 & -5.04 & 63.40 & 59.43 & 6.68 & -1.32 & -7.50 \\
\hline NCEN-3R & 63.55 & 62.75 & 1.27 & -12.10 & -13.21 & 56.65 & 62.80 & -9.79 & -8.48 & 1.45 & 63.00 & 58.45 & 7.78 & 7.60 & -0.17 & 61.07 & 61.33 & -0.42 & -4.95 & -4.54 \\
\hline KDCHH-9810 & 59.90 & 59.60 & 0.50 & -17.15 & -17.57 & 58.95 & 54.65 & 7.87 & -4.77 & -11.71 & 55.80 & 55.40 & 0.72 & -4.70 & -5.38 & 58.22 & 56.55 & 2.95 & -9.39 & -11.98 \\
\hline NCEN-2R & 67.45 & 66.60 & 1.28 & -6.71 & -7.88 & 55.00 & 63.35 & -13.18 & -11.15 & 2.34 & 61.60 & 56.70 & 8.64 & 5.21 & -3.16 & 61.35 & 62.22 & -1.40 & -4.51 & -3.16 \\
\hline JKCH-266 & 54.95 & 56.80 & -3.26 & -24.00 & -21.44 & 58.10 & 62.25 & -6.67 & -6.14 & 0.57 & 62.75 & 59.15 & 6.09 & 7.17 & 1.02 & 58.60 & 59.40 & -1.35 & -8.79 & -7.55 \\
\hline Dhruva & 56.75 & 56.45 & 0.53 & -21.51 & -21.92 & 61.95 & 59.70 & 3.77 & 0.08 & -3.55 & 54.20 & 55.30 & -1.99 & -7.43 & -5.55 & 57.63 & 57.15 & 0.84 & -10.30 & -11.05 \\
\hline K-5316 & 75.55 & 70.30 & 7.47 & 4.50 & -2.77 & 63.30 & 63.30 & 0.00 & 2.26 & 2.26 & 54.75 & 54.15 & 1.11 & -6.49 & -7.51 & 64.53 & 62.58 & 3.12 & 0.44 & -2.60 \\
\hline KDCHH-9632 & 62.10 & 60.17 & 3.21 & -14.11 & -16.78 & 59.40 & 65.95 & -9.93 & -4.04 & 6.54 & 60.75 & 51.65 & 17.62 & 3.76 & -11.78 & 60.75 & 59.26 & 2.51 & -5.45 & -7.77 \\
\hline JK-Varun & 67.00 & 67.45 & -0.67 & -7.33 & -6.71 & 59.75 & 56.30 & 6.13 & -3.47 & -9.05 & 52.30 & 58.25 & -10.21 & -10.67 & -0.51 & 59.68 & 60.67 & -1.63 & -7.11 & -5.57 \\
\hline PCH-2171 & 70.70 & 66.15 & 6.88 & -2.21 & -8.51 & 63.95 & 60.95 & 4.92 & 3.31 & -1.53 & 51.60 & 47.80 & 7.95 & -11.87 & -18.36 & 62.08 & 58.30 & 6.48 & -3.38 & -9.26 \\
\hline JK-Durga & 68.35 & 66.90 & 2.17 & -5.46 & -7.47 & 58.50 & 60.40 & -3.15 & -5.49 & -2.42 & 58.80 & 75.55 & -22.17 & 0.43 & 29.04 & 61.88 & 67.62 & -8.49 & -3.69 & 5.25 \\
\hline JKCH-99 & 65.35 & 61.30 & 6.61 & -9.61 & -15.21 & 64.10 & 61.80 & 3.72 & 3.55 & -0.16 & 63.75 & 65.45 & -2.60 & 8.88 & 11.78 & 64.40 & 62.85 & 2.47 & 0.23 & -2.18 \\
\hline JKCH-1945 & 61.80 & 60.05 & 2.91 & -14.52 & -16.94 & 66.95 & 62.35 & 7.38 & 8.16 & 0.73 & 50.05 & 57.05 & -12.27 & -14.52 & -2.56 & 59.60 & 59.82 & -0.37 & -7.24 & -6.89 \\
\hline JK-Gowri & 61.55 & 57.30 & 7.42 & -14.87 & -20.75 & 62.20 & 59.20 & 5.07 & 0.48 & -4.36 & 54.80 & 56.25 & -2.58 & -6.40 & -3.93 & 59.52 & 57.58 & 3.37 & -7.36 & -10.38 \\
\hline Mean & 65.53 & 63.56 & & & & 61.62 & 61.52 & & & & 57.65 & 56.36 & & & & 61.60 & 60.48 & & & \\
\hline SEm & & & & & & & & & & & & & & & & & & & & \\
\hline CD at $5 \%$ & & & & & & & & & & & & & & & & & & & & \\
\hline CD at $1 \%$ & & & & & & & & & & & & & & & & & & & & \\
\hline
\end{tabular}


Table.3 Mean performance of Bt and non-Bt hybrids across three environments for hull content (\%)

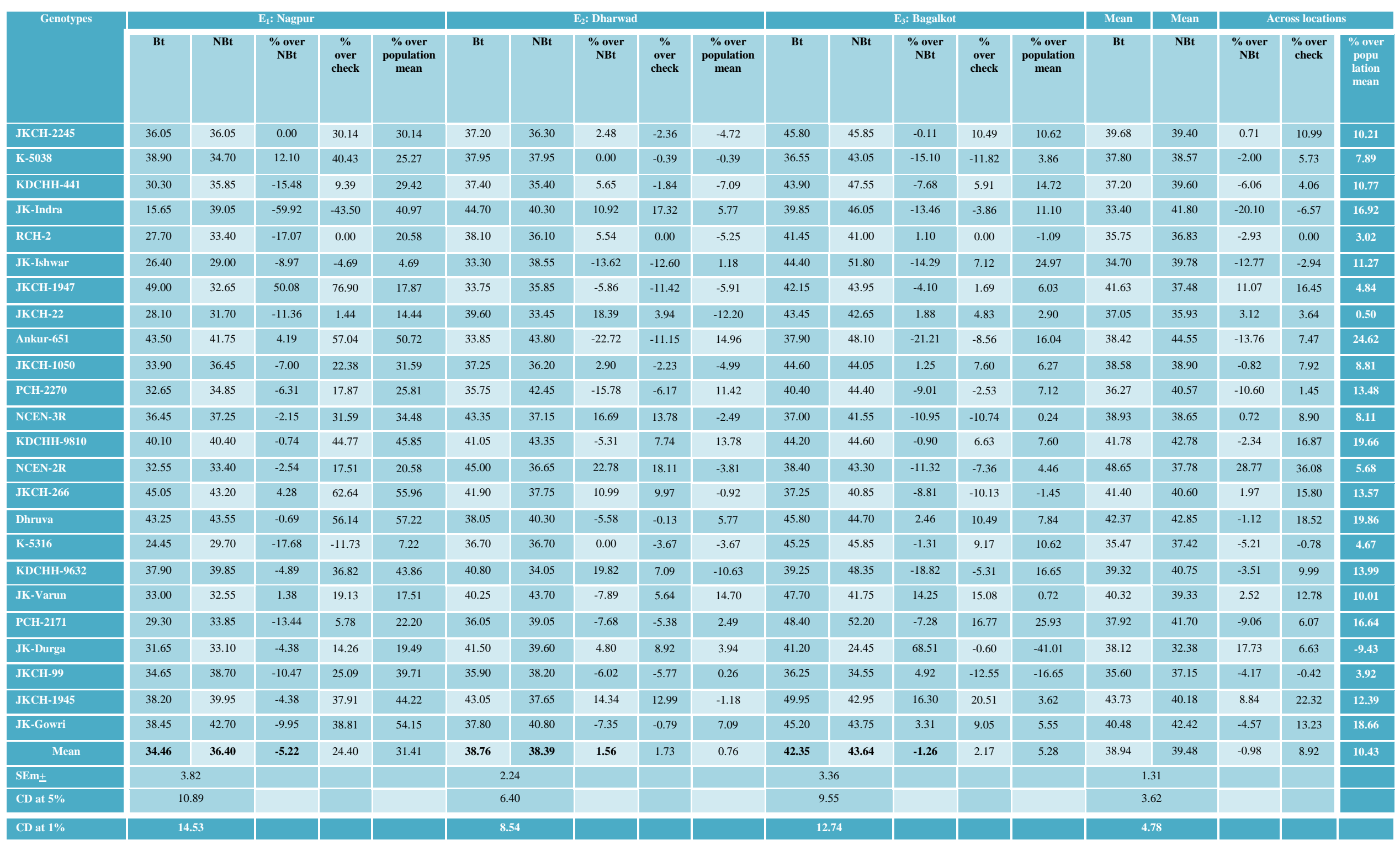


Table.4 Mean performance of Bt and non-Bt hybrids across three environments for ginning outturn (\%)

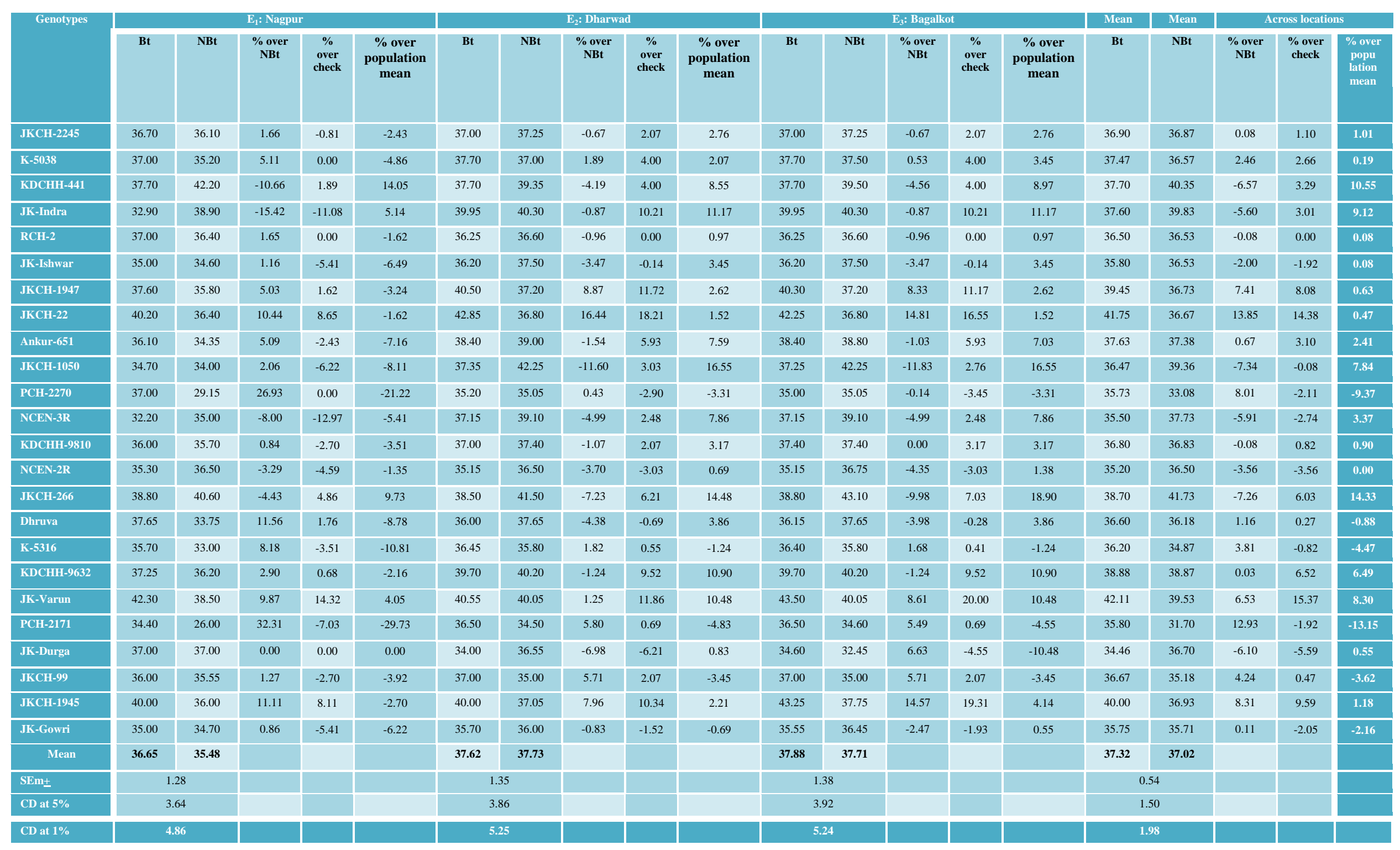


Table.5 Mean performance of Bt and non-Bt hybrids across three environments for seed index (g)

\begin{tabular}{|c|c|c|c|c|c|c|c|c|c|c|c|c|c|c|c|c|c|c|c|c|}
\hline \multirow[t]{2}{*}{ Genotypes } & \multicolumn{5}{|c|}{$E_{1}$ : Nagpur } & \multicolumn{5}{|c|}{$\mathrm{E}_{2}$ : Dharwad } & \multicolumn{5}{|c|}{$\mathrm{E}_{3}$ : Bagalkot } & \multirow{2}{*}{$\begin{array}{c}\text { Mean } \\
\text { Bt }\end{array}$} & \multirow{2}{*}{$\begin{array}{l}\text { Mean } \\
\text { NBt }\end{array}$} & \multicolumn{3}{|c|}{ Across locations } \\
\hline & Bt & NBt & $\begin{array}{c}\text { \% over } \\
\text { NBt }\end{array}$ & $\begin{array}{c}\% \\
\begin{array}{c}\% \\
\text { over } \\
\text { check }\end{array}\end{array}$ & $\begin{array}{c}\% \text { over } \\
\text { population } \\
\text { mean }\end{array}$ & Bt & NBt & $\begin{array}{c}\text { \% over } \\
\text { NBt }\end{array}$ & $\begin{array}{c}\% \\
\begin{array}{c}\% \\
\text { over } \\
\text { check }\end{array}\end{array}$ & $\begin{array}{c}\text { \% over } \\
\text { population } \\
\text { mean }\end{array}$ & $\mathbf{B t}$ & NBt & $\begin{array}{c}\text { \% over } \\
\text { NBt }\end{array}$ & $\begin{array}{c}\% \\
\text { over } \\
\text { check }\end{array}$ & $\begin{array}{c}\text { \% over } \\
\text { population } \\
\text { mean }\end{array}$ & & & $\begin{array}{c}\text { \% over } \\
\text { NBt }\end{array}$ & $\begin{array}{l}\text { \% over } \\
\text { check }\end{array}$ & $\begin{array}{c}\text { \% over } \\
\text { popu } \\
\text { lation } \\
\text { mean }\end{array}$ \\
\hline JKCH-2245 & 8.10 & 8.40 & -3.57 & 9.46 & 13.51 & 8.30 & 9.30 & -10.75 & -5.14 & 6.29 & 6.85 & 8.70 & -21.26 & -2.14 & 24.29 & 7.75 & 8.80 & -11.93 & 0.39 & 13.99 \\
\hline K-5038 & 8.60 & 9.40 & -8.51 & 16.22 & 27.03 & 9.15 & 9.25 & -1.08 & 4.57 & 5.71 & 8.05 & 7.90 & 1.90 & 15.00 & 12.86 & 8.60 & 8.85 & -2.82 & 11.40 & 14.64 \\
\hline KDCHH-441 & 8.60 & 8.00 & 7.50 & 16.22 & 8.11 & 9.60 & 9.45 & 1.59 & 9.71 & 8.00 & 7.15 & 8.50 & -15.88 & 2.14 & 21.43 & 8.45 & 8.65 & -2.31 & 9.46 & 12.05 \\
\hline JK-Indra & 7.90 & 8.00 & -1.25 & 6.76 & 8.11 & 8.95 & 8.90 & 0.56 & 2.29 & 1.71 & 7.10 & 6.55 & 8.40 & 1.43 & -6.43 & 7.98 & 7.82 & 2.05 & 3.37 & 1.30 \\
\hline RCH-2 & 7.40 & 7.80 & -5.13 & 0.00 & 5.41 & 8.75 & 10.30 & -15.05 & 0.00 & 17.71 & 7.00 & 6.85 & 2.19 & 0.00 & -2.14 & 7.72 & 8.32 & -7.21 & 0.00 & 7.77 \\
\hline JK-Ishwar & 8.40 & 9.10 & -7.69 & 13.51 & 22.97 & 7.85 & 10.10 & -22.28 & -10.29 & 15.43 & 7.10 & 7.60 & -6.58 & 1.43 & 8.57 & 7.78 & 8.93 & -12.88 & 0.78 & 15.67 \\
\hline JKCH-1947 & 3.65 & 6.90 & -47.10 & -50.68 & -6.76 & 7.25 & 7.95 & -8.81 & -17.14 & -9.14 & 5.75 & 5.40 & 6.48 & -17.86 & -22.86 & 5.55 & 6.75 & -17.78 & -28.11 & -12.56 \\
\hline JKCH-22 & 5.70 & 7.00 & -18.57 & -22.97 & -5.41 & 7.20 & 8.60 & -16.28 & -17.71 & -1.71 & 6.60 & 9.80 & -32.65 & -5.71 & 40.00 & 6.50 & 8.47 & -23.26 & -15.80 & 9.72 \\
\hline Ankur-651 & 5.50 & 6.00 & -8.33 & -25.68 & -18.92 & 8.95 & 8.65 & 3.47 & 2.29 & -1.14 & 8.55 & 6.20 & 37.90 & 22.14 & -11.43 & 7.67 & 6.95 & 10.36 & -0.65 & -9.97 \\
\hline JKCH-1050 & 5.00 & 5.40 & -7.41 & -32.43 & -27.03 & 8.50 & 7.00 & 21.43 & -2.86 & -20.00 & 6.75 & 6.65 & 1.50 & -3.57 & -5.00 & 6.75 & 6.35 & 6.30 & -12.56 & -17.75 \\
\hline PCH-2270 & 6.40 & 7.80 & -17.95 & -13.51 & 5.41 & 9.15 & 9.65 & -5.18 & 4.57 & 10.29 & 7.65 & 7.20 & 6.25 & 9.29 & 2.86 & 7.73 & 8.22 & -5.96 & 0.13 & 6.48 \\
\hline NCEN-3R & 7.70 & 8.90 & -13.48 & 4.05 & 20.27 & 8.30 & 9.90 & -16.16 & -5.14 & 13.14 & 8.30 & 8.40 & -1.19 & 18.57 & 20.00 & 8.10 & 9.07 & -10.69 & 4.92 & 17.49 \\
\hline KDCHH-9810 & 7.70 & 10.10 & -23.76 & 4.05 & 36.49 & 9.65 & 9.45 & 2.12 & 10.29 & 8.00 & 7.85 & 8.05 & -2.48 & 12.14 & 15.00 & 8.40 & 9.20 & -8.70 & 8.81 & 19.17 \\
\hline NCEN-2R & 8.60 & 9.20 & -6.52 & 16.22 & 24.32 & 9.90 & 8.70 & 13.79 & 13.14 & -0.57 & 6.15 & 6.50 & -5.38 & -12.14 & -7.14 & 8.22 & 8.13 & 1.11 & 6.48 & 5.31 \\
\hline JKCH-266 & 8.40 & 5.90 & 42.37 & 13.51 & -20.27 & 8.20 & 7.20 & 13.89 & -6.29 & -17.71 & 6.45 & 8.55 & -24.56 & -7.86 & 22.14 & 7.68 & 7.22 & 6.37 & -0.52 & -6.48 \\
\hline Dhruva & 7.85 & 8.70 & -9.77 & 6.08 & 17.57 & 8.70 & 10.15 & -14.29 & -0.57 & 16.00 & 7.30 & 7.80 & -6.41 & 4.29 & 11.43 & 7.95 & 8.88 & -10.47 & 2.98 & 15.03 \\
\hline K-5316 & 7.40 & 8.80 & -15.91 & 0.00 & 18.92 & 8.15 & 8.50 & -4.12 & -6.86 & -2.86 & 7.35 & 6.45 & 13.95 & 5.00 & -7.86 & 7.63 & 7.92 & -3.66 & -1.17 & 2.59 \\
\hline KDCHH-9632 & 8.50 & 9.05 & -6.08 & 14.86 & 22.30 & 9.90 & 9.50 & 4.21 & 13.14 & 8.57 & 7.65 & 7.35 & 4.08 & 9.29 & 5.00 & 8.68 & 8.63 & 0.58 & 12.44 & 11.79 \\
\hline JK-Varun & 6.25 & 6.40 & -2.34 & -15.54 & -13.51 & 6.45 & 8.85 & -27.12 & -26.29 & 1.14 & 6.15 & 5.85 & 5.13 & -12.14 & -16.43 & 6.28 & 7.03 & -10.67 & -18.65 & -8.94 \\
\hline PCH-2171 & 6.50 & 7.10 & -8.45 & -12.16 & -4.05 & 8.70 & 8.85 & -1.69 & -0.57 & 1.14 & 6.25 & 5.65 & 10.62 & -10.71 & -19.29 & 7.15 & 7.20 & -0.69 & -7.38 & -6.74 \\
\hline $\begin{array}{l}\text { JK-Durga } \\
\end{array}$ & 6.50 & 8.15 & -20.25 & -12.16 & 10.14 & 8.35 & 8.95 & -6.70 & -4.57 & 2.29 & 6.15 & 6.35 & -3.15 & -12.14 & -9.29 & 7.00 & 7.82 & -10.49 & -9.33 & 1.30 \\
\hline JKCH-99 & 8.10 & 8.90 & -8.99 & 9.46 & 20.27 & 10.40 & 11.25 & -7.56 & 18.86 & 28.57 & 7.60 & 7.95 & -4.40 & 8.57 & 13.57 & 8.70 & 9.37 & -7.15 & 12.69 & 21.37 \\
\hline JKCH-1945 & 5.30 & 5.50 & -3.64 & -28.38 & -25.68 & 7.50 & 7.80 & -3.85 & -14.29 & -10.86 & 6.15 & 6.55 & -6.11 & -12.14 & -6.43 & 6.32 & 6.62 & -4.53 & -18.13 & -14.25 \\
\hline JK-Gowri & 7.50 & 8.80 & -14.77 & 1.35 & 18.92 & 9.45 & 9.25 & 2.16 & 8.00 & 5.71 & 5.80 & 7.60 & -23.68 & -17.14 & 8.57 & 7.58 & 8.55 & -11.35 & -1.81 & 10.75 \\
\hline Mean & 7.15 & 7.89 & & & & 8.64 & 9.06 & & & & 6.99 & 7.27 & & & & 7.59 & 8.07 & & & \\
\hline SEm \pm & & & & & & & & & & & & & & & & & & & & \\
\hline CD at 5\% & & & & & & & & & & & & & & & & & & & & \\
\hline $\mathrm{CD}$ at $1 \%$ & & & & & & & & & & & & & & & & & & & & \\
\hline
\end{tabular}


Table.6 Mean performance of Bt and non-Bt hybrids across three environments for seed cotton yield ( $\mathrm{kg} / \mathrm{ha})$

\begin{tabular}{|c|c|c|c|c|c|c|c|c|c|c|c|c|c|c|c|c|c|c|c|c|}
\hline \multirow[t]{2}{*}{ Genotypes } & \multicolumn{5}{|c|}{$E_{1}:$ Nagpur } & \multicolumn{5}{|c|}{$\mathrm{E}_{2}$ : Dharwad } & \multicolumn{5}{|c|}{$E_{3}:$ Bagalkot } & \multirow{2}{*}{$\begin{array}{c}\text { Mean } \\
\text { Bt }\end{array}$} & \multirow{2}{*}{ Mean } & \multicolumn{3}{|c|}{ Across locations } \\
\hline & Bt & NBt & $\begin{array}{c}\text { \% over } \\
\text { NBt }\end{array}$ & $\begin{array}{l}\text { \% over } \\
\text { check }\end{array}$ & $\begin{array}{c}\% \text { over } \\
\text { population } \\
\text { mean }\end{array}$ & Bt & NBt & $\begin{array}{c}\% \\
\text { over } \\
\text { NBt }\end{array}$ & $\begin{array}{c}\% \\
\text { over } \\
\text { check }\end{array}$ & $\begin{array}{c}\% \text { over } \\
\text { population } \\
\text { mean }\end{array}$ & Bt & NBt & $\begin{array}{c}\text { \% over } \\
\text { NBt }\end{array}$ & $\begin{array}{c}\% \\
\text { over } \\
\text { check }\end{array}$ & $\begin{array}{c}\% \text { over } \\
\text { population } \\
\text { mean }\end{array}$ & & & $\begin{array}{c}\text { \% over } \\
\text { NBBt }\end{array}$ & $\begin{array}{c}\% \\
\text { over } \\
\text { check }\end{array}$ & $\begin{array}{c}\% \\
\text { over } \\
\text { popu } \\
\text { lation } \\
\text { mean }\end{array}$ \\
\hline JKCH-2245 & 2700.00 & 1500.00 & 80.00 & 170.00 & 50.00 & 1793.00 & 1615.00 & 11.02 & 6.41 & -4.15 & 2230.00 & 1703.00 & 30.95 & 161.43 & 99.65 & 2181.67 & 1665.33 & 31.01 & 85.78 & 41.81 \\
\hline K-5038 & 2175.00 & 2000.00 & 8.75 & 117.50 & 100.00 & 1920.00 & 1376.00 & 39.53 & 13.95 & -18.34 & 1541.00 & 998.00 & 54.41 & 80.66 & 17.00 & 1820.33 & 1516.33 & 20.05 & 55.01 & 29.12 \\
\hline КDCHН-441 & 1600.00 & 1120.00 & 42.86 & 60.00 & 12.00 & 1801.00 & 1728.00 & 4.22 & 6.88 & 2.55 & 1625.00 & 1216.00 & 33.63 & 90.50 & 42.56 & 1651.00 & 1379.00 & 19.72 & 40.59 & 17.43 \\
\hline JK-Indra & 2400.00 & 2050.00 & 17.07 & 140.00 & 105.00 & 1615.50 & 1589.00 & 1.67 & -4.12 & -5.70 & 2047.00 & 1213.00 & 68.76 & 139.98 & 42.20 & 2012.00 & 1626.00 & 23.74 & 71.33 & 38.46 \\
\hline RCH-2 & 1000.00 & 650.00 & 53.85 & 0.00 & -35.00 & 1685.00 & 1222.50 & 37.83 & 0.00 & -27.45 & 853.00 & 838.00 & 1.79 & 0.00 & -1.76 & 1174.33 & 908.33 & 29.28 & 0.00 & $\begin{array}{c}- \\
22.65\end{array}$ \\
\hline JK-Ishwar & 1075.00 & 525.00 & 104.76 & 7.50 & -47.50 & 1793.00 & 1583.00 & 13.27 & 6.41 & -6.05 & 1056.00 & 686.00 & 53.94 & 23.80 & -19.58 & 1308.00 & 931.33 & 40.44 & 11.38 & 20.69 \\
\hline JKCH-1947 & 2158.00 & 550.00 & 292.36 & 115.80 & -45.00 & 1446.00 & 1425.00 & 1.47 & $\begin{array}{c}- \\
14.18\end{array}$ & -15.43 & 836.00 & 733.00 & 14.05 & -1.99 & -14.07 & 1480.00 & 902.67 & 63.96 & 26.03 & 23.13 \\
\hline JKCH-22 & 1575.00 & 1150.00 & 36.96 & 57.50 & 15.00 & 2020.00 & 1499.50 & 34.71 & 19.88 & -11.01 & 1677.50 & 1093.00 & 53.48 & 96.66 & 28.14 & 1583.67 & 1421.00 & 11.45 & 34.86 & 21.01 \\
\hline Ankur-651 & 2025.00 & 1350.00 & 50.00 & 102.50 & 35.00 & 2123.00 & 1435.00 & 47.94 & 25.99 & -14.84 & 1146.00 & 1076.00 & 6.51 & 34.35 & 26.14 & 1764.67 & 1287.00 & 37.11 & 50.27 & 9.59 \\
\hline JKCH-1050 & 1165.00 & 525.00 & 121.90 & 16.50 & -47.50 & 1516.00 & 1515.50 & 0.03 & $\begin{array}{c}- \\
10.03\end{array}$ & -10.06 & 1083.00 & 1076.00 & 0.65 & 26.96 & 26.14 & 1252.33 & 1041.00 & 20.30 & 6.64 & 11.35 \\
\hline PCH-2270 & 970.00 & 625.00 & 55.20 & -3.00 & -37.50 & 1441.00 & 1298.00 & 11.02 & $\begin{array}{c}- \\
14.48\end{array}$ & -22.97 & 2061.00 & 1127.00 & 82.87 & 141.62 & 32.12 & 1375.67 & 1131.67 & 21.56 & 17.15 & -3.63 \\
\hline NCEN-3R & 2125.00 & 1175.00 & 80.85 & 112.50 & 17.50 & 1752.00 & 985.00 & 77.87 & 3.98 & -41.54 & 2973.00 & 1020.10 & 191.44 & 248.53 & 19.59 & 2283.33 & 1060.00 & 115.41 & 94.44 & -9.74 \\
\hline KDCHH-9810 & 1950.00 & 1775.00 & 9.86 & 95.00 & 77.50 & 1738.00 & 1091.00 & 59.30 & 3.15 & -35.25 & 1352.00 & 1201.00 & 12.57 & 58.50 & 40.80 & 1680.00 & 1355.67 & 23.92 & 43.06 & 15.44 \\
\hline NCEN-2R & 1950.00 & 1825.00 & 6.85 & 95.00 & 82.50 & 2005.00 & 1703.00 & 17.73 & 18.99 & 1.07 & 1637.00 & 1063.00 & 54.00 & 91.91 & 24.62 & 1763.33 & 1631.00 & 8.11 & 50.16 & 38.89 \\
\hline JKCH-266 & 1550.00 & 1400.00 & 10.71 & 55.00 & 40.00 & 1870.00 & 1420.00 & 31.69 & 10.98 & -15.73 & 1236.00 & 711.00 & 73.84 & 44.90 & -16.65 & 1552.00 & 1177.00 & 31.86 & 32.16 & 0.23 \\
\hline Dhruva & 2150.00 & 1450.00 & 48.28 & 115.00 & 45.00 & 2243.00 & 1748.00 & 28.32 & 33.12 & 3.74 & 1595.00 & 1236.00 & 29.05 & 86.99 & 44.90 & 1831.00 & 1643.00 & 11.44 & 55.92 & 39.91 \\
\hline K-5316 & 1950.00 & 870.00 & 124.14 & 95.00 & -13.00 & 1245.00 & 1116.00 & 11.56 & ${ }^{-}$ & -33.77 & 895.00 & 704.00 & 27.13 & 4.92 & -17.47 & 1256.67 & 1003.33 & 25.25 & 7.01 & 14.56 \\
\hline КDCHH-9632 & 1775.00 & 1600.00 & 10.94 & 77.50 & 60.00 & 1705.00 & 1163.00 & 46.60 & 1.19 & -30.98 & 1728.50 & 634.00 & 172.63 & 102.64 & -25.67 & 1555.33 & 1313.00 & 18.46 & 32.44 & 11.81 \\
\hline JK-Varun & 750.00 & 625.00 & 20.00 & -25.00 & -37.50 & 2086.00 & 1229.00 & 69.73 & 23.80 & -27.06 & 1268.50 & 817.00 & 55.26 & 48.71 & -4.22 & 1326.33 & 932.00 & 42.31 & 12.94 & 20.64 \\
\hline PCH-2171 & 685.00 & 450.00 & 52.22 & -31.50 & -55.00 & 1896.50 & 1440.00 & 31.70 & 12.55 & -14.54 & 1671.50 & 1270.00 & 31.61 & 95.96 & 48.89 & 1339.17 & 1131.67 & 18.34 & 14.04 & -3.63 \\
\hline JK-Durga & 1600.00 & 1350.00 & 18.52 & 60.00 & 35.00 & 1292.00 & 1147.00 & 12.64 & 23.32 & -31.93 & 1150.00 & 549.00 & 109.47 & 34.82 & -35.64 & 1299.00 & 1063.67 & 22.12 & 10.62 & -9.42 \\
\hline JKCH-99 & 1400.00 & 900.00 & 55.56 & 40.00 & -10.00 & 1691.00 & 1486.50 & 13.76 & 0.36 & -11.78 & 1641.00 & 682.00 & 140.62 & 92.38 & -20.05 & 1577.33 & 1022.83 & 54.21 & 34.32 & 12.90 \\
\hline JKCH-1945 & 1250.00 & 1200.00 & 4.17 & 25.00 & 20.00 & 1993.00 & 1760.00 & 13.24 & 18.28 & 4.45 & 2327.00 & 934.00 & 149.14 & 172.80 & 9.50 & 1762.33 & 1392.33 & 26.57 & 50.07 & 18.56 \\
\hline JK-Gowri & 1050.00 & 1000.00 & 5.00 & 5.00 & 0.00 & 1618.00 & 1422.00 & 13.78 & -3.98 & -15.61 & 1443.50 & 850.00 & 69.82 & 69.23 & -0.35 & 1305.00 & 1156.00 & 12.89 & 11.13 & -1.56 \\
\hline Mean & 1626.17 & 1152.71 & & & & 1762.00 & 1416.54 & & & & 1544.73 & 976.25 & & & & 1588.94 & 1237.13 & & & \\
\hline SEm \pm & \multicolumn{2}{|c|}{112.73} & & & & \multicolumn{2}{|c|}{159.79} & & & & \multicolumn{2}{|c|}{146.06} & & & & \multicolumn{2}{|c|}{57.51} & & & \\
\hline CD at $5 \%$ & \multicolumn{2}{|c|}{320.66} & & & & \multicolumn{2}{|c|}{454.54} & & & & \multicolumn{2}{|c|}{415.50} & & & & \multicolumn{2}{|c|}{159.40} & & & \\
\hline CD at $1 \%$ & \multicolumn{2}{|c|}{427.91} & & & & \multicolumn{2}{|c|}{606.56} & & & & \multicolumn{2}{|c|}{554.40} & & & & \multicolumn{2}{|c|}{209.90} & & & \\
\hline
\end{tabular}


Table.7 Mean performance of Bt and non-Bt hybrids across three environments for number of sympodia

\begin{tabular}{|c|c|c|c|c|c|c|c|c|c|c|c|c|c|c|c|c|c|c|c|c|}
\hline \multirow[t]{2}{*}{ Genotypes } & \multicolumn{5}{|c|}{$E_{1}$ : Nagpur } & \multicolumn{5}{|c|}{$E_{2}:$ Dharwad } & \multicolumn{5}{|c|}{$\mathrm{E}_{3}$ : Bagalkot } & \multirow{2}{*}{$\begin{array}{l}\text { Mean } \\
\text { Bt }\end{array}$} & \multirow{2}{*}{$\begin{array}{l}\text { Mean } \\
\text { NBt }\end{array}$} & \multicolumn{3}{|c|}{ Across locations } \\
\hline & Bt & NBt & $\begin{array}{c}\text { \% over } \\
\text { NBt }\end{array}$ & $\begin{array}{c}\% \\
\text { over } \\
\text { check }\end{array}$ & $\begin{array}{c}\% \text { over } \\
\text { population } \\
\text { mean }\end{array}$ & Bt & $\mathrm{NBt}$ & $\begin{array}{c}\text { \% over } \\
\text { NBt }\end{array}$ & $\begin{array}{c}\% \\
\text { over } \\
\text { check }\end{array}$ & $\begin{array}{c}\text { \% over } \\
\text { population } \\
\text { mean }\end{array}$ & Bt & NBt & $\begin{array}{c}\text { \% over } \\
\text { NBt }\end{array}$ & $\begin{array}{c}\% \\
\text { over } \\
\text { check }\end{array}$ & $\begin{array}{c}\text { \% over } \\
\text { population } \\
\text { mean }\end{array}$ & & & $\begin{array}{c}\text { \% over } \\
\text { NBt }\end{array}$ & $\begin{array}{l}\text { \% over } \\
\text { check }\end{array}$ & $\begin{array}{c}\text { \% over } \\
\text { popu } \\
\text { lation } \\
\text { mean }\end{array}$ \\
\hline JKCH-2245 & 16.50 & 12.95 & 27.41 & 37.50 & 7.92 & 13.30 & 11.70 & 13.68 & -6.34 & -17.61 & 13.40 & 14.60 & -8.22 & -18.79 & -11.52 & 14.40 & 13.08 & 10.09 & 1.19 & $\begin{array}{l}-8.08 \\
\end{array}$ \\
\hline K-5038 & 16.10 & 14.50 & 11.03 & 34.17 & 20.83 & 12.60 & 10.30 & 22.33 & -11.27 & -27.46 & 13.20 & 15.30 & -13.73 & -20.00 & -7.27 & 13.97 & 13.37 & 4.49 & -1.83 & -6.04 \\
\hline KDCHH-441 & 14.30 & 12.30 & 16.26 & 19.17 & 2.50 & 14.50 & 13.00 & 11.54 & 2.11 & -8.45 & 15.70 & 15.70 & 0.00 & -4.85 & -4.85 & 14.83 & 13.67 & 8.49 & 4.22 & -3.94 \\
\hline JK-Indra & 14.70 & 13.10 & 12.21 & 22.50 & 9.17 & 13.50 & 12.60 & 7.14 & -4.93 & -11.27 & 17.40 & 15.30 & 13.73 & 5.45 & -7.27 & 15.20 & 13.67 & 11.19 & 6.82 & -3.94 \\
\hline RCH-2 & 12.00 & 12.30 & -2.44 & 0.00 & 2.50 & 14.20 & 11.50 & 23.48 & 0.00 & -19.01 & 16.50 & 15.90 & 3.77 & 0.00 & -3.64 & 14.23 & 13.23 & 7.56 & 0.00 & -7.03 \\
\hline JK-Ishwar & 13.20 & 10.15 & 30.05 & 10.00 & -15.42 & 14.40 & 12.30 & 17.07 & 1.41 & -13.38 & 14.20 & 15.80 & -10.13 & -13.94 & -4.24 & 13.93 & 12.75 & 9.25 & -2.11 & -10.40 \\
\hline JKCH-1947 & 13.00 & 12.50 & 4.00 & 8.33 & 4.17 & 14.70 & 16.30 & -9.82 & 3.52 & 14.79 & 16.00 & 14.60 & 9.59 & -3.03 & -11.52 & 14.57 & 14.47 & 0.69 & 2.39 & 1.69 \\
\hline JKCH-22 & 11.80 & 11.30 & 4.42 & -1.67 & -5.83 & 12.40 & 13.40 & -7.46 & -12.68 & -5.63 & 15.20 & 17.70 & -14.12 & -7.88 & 7.27 & 13.13 & 14.13 & -7.08 & -7.73 & -0.70 \\
\hline Ankur-651 & 15.40 & 16.20 & -4.94 & 28.33 & 35.00 & 14.30 & 13.10 & 9.16 & 0.70 & -7.75 & 15.90 & 13.70 & 16.06 & -3.64 & -16.97 & 15.20 & 14.33 & 6.07 & 6.82 & 0.70 \\
\hline JKCH-1050 & 14.50 & 12.70 & 14.17 & 20.83 & 5.83 & 14.70 & 14.60 & 0.68 & 3.52 & 2.82 & 13.40 & 10.30 & 30.10 & -18.79 & -37.58 & 14.20 & 12.53 & 13.33 & -0.21 & -11.95 \\
\hline PCH-2270 & 12.60 & 13.30 & -5.26 & 5.00 & 10.83 & 13.90 & 13.00 & 6.92 & -2.11 & -8.45 & 14.90 & 19.80 & -24.75 & -9.70 & 20.00 & 13.80 & 15.37 & -10.21 & -3.02 & 8.01 \\
\hline NCEN-3R & 15.10 & 16.10 & -6.21 & 25.83 & 34.17 & 13.80 & 13.10 & 5.34 & -2.82 & -7.75 & 12.40 & 14.50 & -14.48 & -24.85 & -12.12 & 13.77 & 14.57 & -5.49 & -3.23 & 2.39 \\
\hline KDCHH-9810 & 14.80 & 14.00 & 5.71 & 23.33 & 16.67 & 14.20 & 14.50 & -2.07 & 0.00 & 2.11 & 17.30 & 11.20 & 54.46 & 4.85 & -32.12 & 15.43 & 13.23 & 16.63 & 8.43 & -7.03 \\
\hline NCEN-2R & 12.50 & 14.50 & -13.79 & 4.17 & 20.83 & 13.00 & 13.40 & -2.99 & -8.45 & -5.63 & 15.40 & 12.60 & 22.22 & -6.67 & -23.64 & 13.63 & 13.50 & 0.96 & -4.22 & -5.13 \\
\hline JKCH-266 & 12.90 & 13.20 & -2.27 & 7.50 & 10.00 & 13.70 & 13.20 & 3.79 & -3.52 & -7.04 & 14.60 & 14.20 & 2.82 & -11.52 & -13.94 & 13.73 & 13.53 & 1.48 & -3.51 & $\begin{array}{l}-4.92 \\
\end{array}$ \\
\hline Dhruva & 16.20 & 14.80 & 9.46 & 35.00 & 23.33 & 13.20 & 12.90 & 2.33 & -7.04 & -9.15 & 13.90 & 14.30 & -2.80 & -15.76 & -13.33 & 14.43 & 14.00 & 3.07 & 1.41 & -1.62 \\
\hline K-5316 & 16.10 & 15.50 & 3.87 & 34.17 & 29.17 & 13.30 & 13.30 & 0.00 & -6.34 & -6.34 & 11.30 & 12.20 & -7.38 & -31.52 & -26.06 & 13.57 & 13.67 & -0.73 & -4.64 & -3.94 \\
\hline КDCHH-9632 & 13.60 & 13.20 & 3.03 & 13.33 & 10.00 & 14.00 & 12.30 & 13.82 & -1.41 & -13.38 & 17.40 & 21.50 & -19.07 & 5.45 & 30.30 & 15.00 & 15.67 & -4.28 & 5.41 & 10.12 \\
\hline JK-Varun & 30.50 & 12.20 & 150.00 & 154.17 & 1.67 & 15.70 & 11.00 & 42.73 & 10.56 & -22.54 & 13.00 & 14.70 & -11.56 & -21.21 & -10.91 & 19.73 & 12.63 & 56.22 & 38.65 & \begin{tabular}{l|l|}
-11.24 \\
\end{tabular} \\
\hline PCH-2171 & 15.40 & 12.60 & 22.22 & 28.33 & 5.00 & 13.50 & 13.70 & -1.46 & -4.93 & -3.52 & 11.90 & 15.00 & -20.67 & -27.88 & -9.09 & 13.60 & 13.77 & -1.23 & -4.43 & -3.23 \\
\hline JK-Durga & 13.50 & 12.80 & 5.47 & 12.50 & 6.67 & 13.60 & 13.00 & 4.62 & -4.23 & -8.45 & 14.00 & 15.70 & -10.83 & -15.15 & -4.85 & 13.70 & 13.83 & -0.94 & -3.72 & -2.81 \\
\hline JKCH-99 & 15.60 & 15.80 & -1.27 & 30.00 & 31.67 & 14.50 & 11.90 & 21.85 & 2.11 & -16.20 & 18.50 & 18.30 & 1.09 & 12.12 & 10.91 & 16.20 & 15.33 & 5.68 & 13.84 & 7.73 \\
\hline JKCH-1945 & 13.60 & 13.20 & 3.03 & 13.33 & 10.00 & 13.90 & 13.70 & 1.46 & -2.11 & -3.52 & 15.00 & 14.50 & 3.45 & -9.09 & -12.12 & 14.17 & 13.80 & 2.68 & -0.42 & -3.02 \\
\hline JK-Gowri & 12.30 & 12.75 & -3.53 & 2.50 & 6.25 & 11.50 & 11.70 & -1.71 & -19.01 & -17.61 & 14.05 & 14.90 & -5.70 & -14.85 & -9.70 & 12.62 & 13.12 & -3.81 & -11.31 & -7.80 \\
\hline Mean & 14.84 & 13.41 & & & & 13.77 & 12.90 & & & & 14.77 & 15.10 & & & & 14.46 & 13.80 & & & \\
\hline SEm \pm & \multicolumn{2}{|c|}{0.91} & & & & \multicolumn{2}{|c|}{0.76} & & & & \multicolumn{2}{|c|}{1.45} & & & & \multicolumn{2}{|c|}{0.44} & & & \\
\hline CD at $5 \%$ & \multicolumn{2}{|c|}{2.61} & & & & \multicolumn{2}{|c|}{2.17} & & & & & & & & & \multicolumn{2}{|c|}{1.22} & & & \\
\hline CD at $1 \%$ & \multicolumn{2}{|c|}{3.48} & & & & \multicolumn{2}{|c|}{2.89} & & & & \multicolumn{2}{|c|}{5.51} & & & & \multicolumn{2}{|c|}{1.61} & & & \\
\hline
\end{tabular}


Table.8 Mean performance of Bt and non-Bt hybrids across three environments for bolls per plant

\begin{tabular}{|c|c|c|c|c|c|c|c|c|c|c|c|c|c|c|c|c|c|c|c|c|}
\hline \multirow[t]{2}{*}{ Genotypes } & \multicolumn{5}{|c|}{$E_{1}:$ Nagpur } & \multicolumn{5}{|c|}{$\mathrm{E}_{2}$ : Dharwad } & \multicolumn{5}{|c|}{$\mathrm{E}_{3}$ : Bagalkot } & \multirow{2}{*}{$\begin{array}{c}\text { Mean } \\
\text { Bt }\end{array}$} & \multirow{2}{*}{$\begin{array}{l}\text { Mean } \\
\text { NBt }\end{array}$} & \multicolumn{3}{|c|}{ Across locations } \\
\hline & Bt & NBt & $\begin{array}{c}\text { \% over } \\
\text { NBt }\end{array}$ & $\begin{array}{c}\begin{array}{c}\% \\
\text { over } \\
\text { check }\end{array} \\
\end{array}$ & $\begin{array}{c}\text { \% over } \\
\text { population } \\
\text { mean }\end{array}$ & Bt & NBt & $\begin{array}{c}\text { \% over } \\
\text { NBt }\end{array}$ & $\begin{array}{c}\% \\
\begin{array}{c}\% \\
\text { over } \\
\text { check }\end{array}\end{array}$ & $\begin{array}{c}\% \text { over } \\
\text { population } \\
\text { mean }\end{array}$ & Bt & NBt & $\begin{array}{c}\text { \% over } \\
\text { NBt }\end{array}$ & $\begin{array}{c}\% \\
\text { over } \\
\text { check }\end{array}$ & $\begin{array}{c}\% \text { over } \\
\text { population } \\
\text { mean }\end{array}$ & & & $\begin{array}{c}\text { \% over } \\
\text { NBt }\end{array}$ & $\begin{array}{c}\% \\
\begin{array}{c}\% \\
\text { over } \\
\text { check }\end{array}\end{array}$ & $\begin{array}{c}\% \\
\text { over } \\
\text { popu } \\
\text { lation } \\
\text { mean }\end{array}$ \\
\hline JKCH-2245 & 28.50 & 24.50 & 16.33 & 36.36 & 17.22 & 29.30 & 24.20 & 21.07 & 10.98 & -8.33 & 31.90 & 17.00 & 87.65 & -12.84 & -53.55 & 28.57 & 23.23 & 22.99 & 4.92 & -14.69 \\
\hline K-5038 & 25.50 & 23.00 & 10.87 & 22.01 & 10.05 & 23.40 & 22.80 & 2.63 & -11.36 & -13.64 & 31.50 & 30.60 & 2.94 & -13.93 & -16.39 & 26.30 & 25.97 & 1.27 & -3.42 & -4.63 \\
\hline КDСНН-441 & 32.50 & 25.00 & 30.00 & 55.50 & 19.62 & 28.15 & 27.70 & 1.62 & 6.63 & 4.92 & 37.00 & 33.60 & 10.12 & 1.09 & -8.20 & 32.55 & 28.77 & 13.14 & 19.54 & 5.66 \\
\hline JK-Indra & 22.40 & 20.00 & 12.00 & 7.18 & -4.31 & 25.20 & 21.60 & 16.67 & -4.55 & -18.18 & 40.00 & 32.00 & 25.00 & 9.29 & -12.57 & 28.00 & 25.73 & 8.82 & 2.83 & -5.51 \\
\hline RCH-2 & 20.90 & 19.10 & 9.42 & 0.00 & -8.61 & 26.40 & 26.00 & 1.54 & 0.00 & -1.52 & 36.60 & 33.00 & 10.91 & 0.00 & -9.84 & 27.23 & 26.77 & 1.72 & 0.00 & -1.69 \\
\hline JK-Ishwar & 31.60 & 15.50 & 103.87 & 51.20 & -25.84 & 22.80 & 22.50 & 1.33 & -13.64 & -14.77 & 34.89 & 29.70 & 17.47 & -4.67 & -18.85 & 27.93 & 24.40 & 14.47 & 2.57 & -10.39 \\
\hline JKCH-1947 & 28.50 & 27.00 & 5.56 & 36.36 & 29.19 & 23.50 & 19.30 & 21.76 & -10.98 & -26.89 & 28.00 & 25.10 & 11.55 & -23.50 & -31.42 & 26.17 & 24.30 & 7.70 & -3.89 & -10.76 \\
\hline JKCH-22 & 24.50 & 13.50 & 81.48 & 17.22 & -35.41 & 26.60 & 22.50 & 18.22 & 0.76 & -14.77 & 31.00 & 29.00 & 6.90 & -15.30 & -20.77 & 27.37 & 21.67 & 26.30 & 0.51 & -20.42 \\
\hline Ankur-651 & 25.00 & 25.00 & 0.00 & 19.62 & 19.62 & 27.00 & 24.00 & 12.50 & 2.27 & -9.09 & 34.10 & 27.00 & 26.30 & -6.83 & -26.23 & 28.70 & 25.33 & 13.30 & 5.40 & -6.98 \\
\hline JKCH-1050 & 23.50 & 16.85 & 39.47 & 12.44 & -19.38 & 26.60 & 20.00 & 33.00 & 0.76 & -24.24 & 37.10 & 32.60 & 13.80 & 1.37 & -10.93 & 29.07 & 23.15 & 25.57 & 6.76 & -14.98 \\
\hline PCH-2270 & 25.00 & 16.00 & 56.25 & 19.62 & -23.44 & 27.40 & 22.00 & 24.55 & 3.79 & -16.67 & 38.20 & 32.50 & 17.54 & 4.37 & -11.20 & 28.30 & 25.40 & 11.42 & 3.93 & -6.72 \\
\hline NCEN-3R & 29.70 & 22.00 & 35.00 & 42.11 & 5.26 & 27.80 & 23.30 & 19.31 & 5.30 & -11.74 & 32.50 & 30.00 & 8.33 & -11.20 & -18.03 & 30.00 & 25.10 & 19.52 & 10.17 & -7.82 \\
\hline KDCHH-9810 & 32.00 & 26.00 & 23.08 & 53.11 & 24.40 & 29.70 & 27.90 & 6.45 & 12.50 & 5.68 & 23.00 & 23.00 & 0.00 & -37.16 & -37.16 & 28.23 & 25.63 & 10.14 & 3.67 & -5.88 \\
\hline NCEN-2R & 42.00 & 33.40 & 25.75 & 100.96 & 59.81 & 26.10 & 25.90 & 0.77 & -1.14 & -1.89 & 35.80 & 35.00 & 2.29 & -2.19 & -4.37 & 34.57 & 31.50 & 9.75 & 26.96 & 15.68 \\
\hline JKCH-266 & 24.70 & 15.00 & 64.67 & 18.18 & -28.23 & 32.50 & 26.50 & 22.64 & 23.11 & 0.38 & 35.00 & 28.50 & 22.81 & -4.37 & -22.13 & 27.50 & 26.57 & 3.50 & 0.99 & -2.42 \\
\hline Dhruva & 27.00 & 26.00 & 3.85 & 29.19 & 24.40 & 26.50 & 25.50 & 3.92 & 0.38 & -3.41 & 31.30 & 31.00 & 0.97 & -14.48 & -15.30 & 28.17 & 27.60 & 2.07 & 3.45 & 1.36 \\
\hline K-5316 & 23.00 & 22.50 & 2.22 & 10.05 & 7.66 & 20.60 & 18.20 & 13.19 & -21.97 & -31.06 & 35.70 & 26.00 & 37.31 & -2.46 & -28.96 & 26.43 & 22.23 & 18.89 & -2.94 & -18.36 \\
\hline КDCHН-9632 & 33.50 & 20.80 & 61.06 & 60.29 & -0.48 & 23.30 & 23.00 & 1.30 & -11.74 & -12.88 & 42.00 & 33.00 & 27.27 & 14.75 & -9.84 & 32.83 & 25.70 & 27.74 & 20.57 & -5.62 \\
\hline JK-Varun & 25.50 & 20.00 & 27.50 & 22.01 & -4.31 & 34.90 & 29.50 & 18.31 & 32.20 & 11.74 & 36.30 & 33.50 & 8.36 & -0.82 & -8.47 & 32.23 & 27.67 & 16.48 & 18.36 & 1.62 \\
\hline PCH-2171 & 23.40 & 18.15 & 28.93 & 11.96 & -13.16 & 26.00 & 25.10 & 3.59 & -1.52 & -4.92 & 36.10 & 26.40 & 36.74 & -1.37 & -27.87 & 26.75 & 24.97 & 7.13 & -1.76 & -8.30 \\
\hline JK-Durga & 23.40 & 20.00 & 17.00 & 11.96 & -4.31 & 25.40 & 18.20 & 39.56 & -3.79 & -31.06 & 43.60 & 35.00 & 24.57 & 19.13 & -4.37 & 29.67 & 25.53 & 16.22 & 8.96 & -6.24 \\
\hline JKCH-99 & 33.20 & 32.50 & 2.15 & 58.85 & 55.50 & 29.80 & 25.50 & 16.86 & 12.88 & -3.41 & 41.00 & 34.10 & 20.23 & 12.02 & -6.83 & 34.67 & 30.70 & 12.93 & 27.32 & 12.74 \\
\hline JКСН-1945 & 26.50 & 19.50 & 35.90 & 26.79 & -6.70 & 30.20 & 28.20 & 7.09 & 14.39 & 6.82 & 32.10 & 24.80 & 29.44 & -12.30 & -32.24 & 27.17 & 26.60 & 2.14 & -0.22 & -2.31 \\
\hline JK-Gowri & 29.00 & 14.20 & 104.23 & 38.76 & -32.06 & 25.60 & 21.30 & 20.19 & -3.03 & -19.32 & 37.20 & 31.00 & 20.00 & 1.64 & -15.30 & 28.53 & 24.23 & 17.75 & 4.77 & -11.02 \\
\hline Mean & 27.53 & 21.48 & & & & 26.86 & 23.78 & & & & 35.08 & 29.73 & & & & 29.04 & 25.78 & & & \\
\hline SEm \pm & \multicolumn{2}{|c|}{2.38} & & & & \multicolumn{2}{|c|}{2.37} & & & & \multicolumn{2}{|c|}{1.85} & & & & \multicolumn{2}{|c|}{0.90} & & & \\
\hline CD at $5 \%$ & \multicolumn{2}{|c|}{6.78} & & & & \multicolumn{2}{|c|}{6.76} & & & & \multicolumn{2}{|c|}{5.26} & & & & \multicolumn{2}{|c|}{2.50} & & & \\
\hline $\mathrm{CD}$ at $1 \%$ & \multicolumn{2}{|c|}{9.05} & & & & \multicolumn{2}{|c|}{9.01} & & & & \multicolumn{2}{|c|}{7.02} & & & & \multicolumn{2}{|c|}{3.30} & & & \\
\hline
\end{tabular}


Table.9 Mean performance of Bt and non-Bt hybrids across three environments for twenty boll weight (g)

\begin{tabular}{|c|c|c|c|c|c|c|c|c|c|c|c|c|c|c|c|c|c|c|c|c|}
\hline \multirow[t]{2}{*}{ Genotypes } & \multicolumn{5}{|c|}{$\mathrm{E}_{1}$ : Nagpur } & \multicolumn{5}{|c|}{$E_{2}$ : Dharwad } & \multicolumn{5}{|c|}{$E_{3}$ : Bagalkot } & \multirow{2}{*}{$\begin{array}{c}\text { Mean } \\
\text { Bt }\end{array}$} & \multirow{2}{*}{$\begin{array}{l}\text { Mean } \\
\text { NBt }\end{array}$} & \multicolumn{3}{|c|}{ Across locations } \\
\hline & Bt & NBt & $\begin{array}{c}\text { \% over } \\
\text { NBBt }\end{array}$ & $\begin{array}{c}\% \\
\text { over } \\
\text { check }\end{array}$ & $\begin{array}{c}\% \text { over } \\
\text { population } \\
\text { mean }\end{array}$ & Bt & $\mathbf{N B t}$ & $\begin{array}{c}\text { \% over } \\
\mathbf{N B B}\end{array}$ & $\begin{array}{c}\% \\
\text { over } \\
\text { check }\end{array}$ & $\begin{array}{c}\text { \% over } \\
\text { population } \\
\text { mean }\end{array}$ & Bt & NBt & $\begin{array}{c}\text { \% over } \\
\mathbf{N B B}\end{array}$ & $\begin{array}{c}\begin{array}{c}\% \\
\text { over } \\
\text { check }\end{array} \\
\end{array}$ & $\begin{array}{c}\% \text { over } \\
\text { population } \\
\text { mean }\end{array}$ & & & $\begin{array}{c}\text { \% over } \\
\mathbf{N B B}\end{array}$ & $\begin{array}{c}\% \\
\text { over } \\
\text { check }\end{array}$ & $\begin{array}{c}\% \\
\text { over } \\
\text { popu } \\
\text { lation } \\
\text { mean }\end{array}$ \\
\hline JKCH-2245 & 65.00 & 65.00 & 0.00 & -14.47 & -14.47 & 90.50 & 90.00 & 0.56 & -12.14 & -12.62 & 68.00 & 66.00 & 3.03 & -8.11 & -10.81 & 74.50 & 73.67 & 1.13 & -11.66 & -12.64 \\
\hline K-5038 & 90.00 & 85.00 & 5.88 & 18.42 & 11.84 & 100.50 & 99.50 & 1.01 & -2.43 & -3.40 & 68.00 & 67.50 & 0.74 & -8.11 & -8.78 & 85.67 & 84.50 & 1.38 & 1.59 & 0.20 \\
\hline КDCHН-441 & 85.00 & 70.00 & 21.43 & 11.84 & -7.89 & 90.50 & 86.00 & 5.23 & -12.14 & -16.50 & 73.00 & 73.00 & 0.00 & -1.35 & -1.35 & 81.33 & 77.83 & 4.50 & -3.56 & -7.71 \\
\hline JK-Indra & 87.00 & 84.00 & 3.57 & 14.47 & 10.53 & 99.00 & 97.50 & 1.54 & -3.88 & -5.34 & 76.00 & 69.50 & 9.35 & 2.70 & -6.08 & 87.33 & 83.67 & 4.37 & 3.56 & -0.78 \\
\hline RCH-2 & 76.00 & 73.00 & 4.11 & 0.00 & -3.95 & 103.00 & 93.00 & 10.75 & 0.00 & -9.71 & 74.00 & 61.50 & 20.33 & 0.00 & -16.89 & 84.33 & 75.83 & 11.21 & 0.00 & -10.08 \\
\hline JK-Ishwar & 96.00 & 85.00 & 12.94 & 26.32 & 11.84 & 113.00 & 109.50 & 3.20 & 9.71 & 6.31 & 77.00 & 52.50 & 46.67 & 4.05 & -29.05 & 90.50 & 87.17 & 3.82 & 7.32 & 3.37 \\
\hline JKCH-1947 & 71.00 & 59.00 & 20.34 & -6.58 & -22.37 & 103.00 & 96.00 & 7.29 & 0.00 & -6.80 & 68.50 & 65.50 & 4.58 & -7.43 & -11.49 & 80.83 & 73.50 & 9.97 & -4.15 & -12.84 \\
\hline JKCH-22 & 79.00 & 79.00 & 0.00 & 3.95 & 3.95 & 104.00 & 100.50 & 3.48 & 0.97 & -2.43 & 57.50 & 57.00 & 0.88 & -22.30 & -22.97 & 80.00 & 79.00 & 1.27 & -5.13 & -6.32 \\
\hline Ankur-651 & 67.00 & 67.00 & 0.00 & -11.84 & -11.84 & 96.00 & 86.50 & 10.98 & -6.80 & -16.02 & 59.50 & 54.50 & 9.17 & -19.59 & -26.35 & 74.17 & 69.33 & 6.98 & -12.05 & -17.79 \\
\hline JKCH-1050 & 74.00 & 70.00 & 5.71 & -2.63 & -7.89 & 90.00 & 86.50 & 4.05 & -12.62 & -16.02 & 59.00 & 55.50 & 6.31 & -20.27 & -25.00 & 73.00 & 72.00 & 1.39 & -13.44 & -14.62 \\
\hline PCH-2270 & 78.00 & 74.00 & 5.41 & 2.63 & -2.63 & 110.00 & 93.00 & 18.28 & 6.80 & -9.71 & 75.50 & 64.00 & 17.97 & 2.03 & -13.51 & 86.50 & 78.33 & 10.43 & 2.57 & -7.11 \\
\hline NCEN-3R & 98.00 & 77.00 & 27.27 & 28.95 & 1.32 & 108.50 & 95.00 & 14.21 & 5.34 & -7.77 & 70.00 & 66.00 & 6.06 & -5.41 & -10.81 & 90.83 & 80.67 & 12.59 & 7.71 & -4.34 \\
\hline КDCHН-9810 & 90.00 & 67.00 & 34.33 & 18.42 & -11.84 & 127.00 & 109.00 & 16.51 & 23.30 & 5.83 & 83.00 & 74.50 & 11.41 & 12.16 & 0.68 & 92.33 & 91.17 & 1.27 & 9.49 & 8.11 \\
\hline NCEN-2R & 96.00 & 55.00 & 74.55 & 26.32 & -27.63 & 111.50 & 110.50 & 0.90 & 8.25 & 7.28 & 70.50 & 53.00 & 33.02 & -4.73 & -28.38 & 86.50 & 79.00 & 9.49 & 2.57 & -6.32 \\
\hline JKCH-266 & 71.00 & 67.00 & 5.97 & -6.58 & -11.84 & 105.00 & 92.50 & 13.51 & 1.94 & -10.19 & 67.50 & 62.50 & 8.00 & -8.78 & -15.54 & 78.17 & 77.00 & 1.52 & -7.30 & -8.69 \\
\hline Dhruva & 105.20 & 85.00 & 23.76 & 38.42 & 11.84 & 108.00 & 100.50 & 7.46 & 4.85 & -2.43 & 64.00 & 63.50 & 0.79 & -13.51 & -14.19 & 89.90 & 85.50 & 5.15 & 6.61 & 1.39 \\
\hline K-5316 & 110.00 & 92.00 & 19.57 & 44.74 & 21.05 & 95.00 & 93.00 & 2.15 & -7.77 & -9.71 & 70.00 & 69.50 & 0.72 & -5.41 & -6.08 & 91.67 & 84.83 & 8.06 & 8.70 & 0.59 \\
\hline КDCHН-9632 & 92.00 & 77.00 & 19.48 & 21.05 & 1.32 & 95.50 & 88.50 & 7.91 & -7.28 & -14.08 & 69.00 & 62.00 & 11.29 & -6.76 & -16.22 & 83.17 & 78.17 & 6.40 & -1.38 & -7.30 \\
\hline JK-Varun & 64.00 & 51.00 & 25.49 & -15.79 & -32.89 & 89.00 & 80.50 & 10.56 & -13.59 & -21.84 & 62.50 & 55.50 & 12.61 & -15.54 & -25.00 & 69.00 & 65.17 & 5.88 & -18.18 & -22.72 \\
\hline PCH-2171 & 64.00 & 55.00 & 16.36 & -15.79 & -27.63 & 98.00 & 90.50 & 8.29 & -4.85 & -12.14 & 67.50 & 62.50 & 8.00 & -8.78 & -15.54 & 74.00 & 71.83 & 3.02 & -12.25 & -14.82 \\
\hline JK-Durga & 82.00 & 72.00 & 13.89 & 7.89 & -5.26 & 101.00 & 88.50 & 14.12 & -1.94 & -14.08 & 50.00 & 47.00 & 6.38 & -32.43 & -36.49 & 77.67 & 69.17 & 12.29 & -7.90 & -17.98 \\
\hline JKCH-99 & 82.00 & 60.00 & 36.67 & 7.89 & -21.05 & 92.50 & 91.00 & 1.65 & -10.19 & -11.65 & 64.50 & 60.50 & 6.61 & -12.84 & -18.24 & 79.17 & 71.00 & 11.51 & -6.12 & -15.81 \\
\hline JKCH-1945 & 75.00 & 68.00 & 10.29 & -1.32 & -10.53 & 93.50 & 86.00 & 8.72 & -9.22 & -16.50 & 68.50 & 67.00 & 2.24 & -7.43 & -9.46 & 76.50 & 76.17 & 0.43 & -9.28 & -9.68 \\
\hline JK-Gowri & 101.00 & 80.00 & 26.25 & 32.89 & 5.26 & 101.00 & 97.50 & 3.59 & -1.94 & -5.34 & 74.50 & 71.00 & 4.93 & 0.68 & -4.05 & 89.83 & 85.17 & 5.47 & 6.52 & 1.00 \\
\hline Mean & 83.26 & 71.54 & & & & 101.04 & 94.21 & & & & 68.23 & 62.54 & & & & 82.37 & 77.90 & & & \\
\hline $\mathrm{SEm} \pm$ & & & & & & & & & & & & & & & & & & & & \\
\hline CD at $5 \%$ & & & & & & & & & & & & & & & & & & & & \\
\hline CD at $1 \%$ & & & & & & & & & & & & & & & & & & & & \\
\hline
\end{tabular}


Table.10 Mean performance of Bt and non-Bt hybrids across three environments for plant height $(\mathrm{cm})$

\begin{tabular}{|c|c|c|c|c|c|c|c|c|c|c|c|c|c|c|c|c|c|c|c|c|}
\hline \multirow[t]{2}{*}{ Genotypes } & \multicolumn{5}{|c|}{$E_{1}:$ Nagpur } & \multicolumn{5}{|c|}{$\mathrm{E}_{2}$ : Dharwad } & \multicolumn{5}{|c|}{$E_{3}$ : Bagalkot } & \multirow{2}{*}{$\begin{array}{c}\text { Mean } \\
\text { Bt }\end{array}$} & \multirow{2}{*}{$\begin{array}{l}\text { Mean } \\
\text { NBt }\end{array}$} & \multicolumn{3}{|c|}{ Across locations } \\
\hline & Bt & NBt & $\begin{array}{c}\text { \% over } \\
\mathbf{N B t}\end{array}$ & $\begin{array}{l}\text { \% over } \\
\text { check }\end{array}$ & $\begin{array}{c}\% \text { over } \\
\text { population } \\
\text { mean }\end{array}$ & $\mathbf{B t}$ & NBt & $\begin{array}{c}\text { \% over } \\
\mathrm{NBt}\end{array}$ & $\begin{array}{c}\text { \% over } \\
\text { check }\end{array}$ & $\begin{array}{c}\% \text { over } \\
\text { population } \\
\text { mean }\end{array}$ & Bt & NBt & $\begin{array}{c}\text { \% over } \\
\text { NBt }\end{array}$ & $\begin{array}{c}\text { \% over } \\
\text { check }\end{array}$ & $\begin{array}{c}\% \text { over } \\
\text { population } \\
\text { mean }\end{array}$ & & & $\begin{array}{c}\text { \% over } \\
\text { NBt }\end{array}$ & $\begin{array}{l}\text { \% over } \\
\text { check }\end{array}$ & $\begin{array}{c}\text { \% over } \\
\text { popu } \\
\text { lation } \\
\text { mean }\end{array}$ \\
\hline JKCH-2245 & 91.60 & 96.00 & -4.58 & 66.55 & 74.55 & 83.05 & 104.30 & -20.37 & -4.65 & 89.64 & 98.70 & 98.43 & 0.27 & -20.56 & 78.96 & 91.12 & 91.60 & -0.52 & 2.64 & 66.55 \\
\hline K-5038 & 93.00 & 87.30 & 6.53 & 69.09 & 58.73 & 71.80 & 120.40 & -40.37 & -17.57 & 118.91 & 119.30 & 100.93 & 18.20 & -3.98 & 83.51 & 94.70 & 93.00 & 1.83 & 6.67 & 69.09 \\
\hline КDCHН-441 & 89.95 & 82.50 & 9.03 & 63.55 & 50.00 & 90.55 & 105.70 & -14.33 & 3.96 & 92.18 & 111.00 & 89.87 & 23.51 & -10.66 & 63.40 & 97.17 & 89.95 & 8.03 & 9.45 & 63.55 \\
\hline JK-Indra & 80.90 & 93.30 & -13.29 & 47.09 & 69.64 & 83.30 & 98.70 & -15.60 & -4.36 & 79.45 & 99.50 & 95.47 & 4.22 & -19.92 & 73.58 & 87.90 & 80.90 & 8.65 & -0.99 & 47.09 \\
\hline RCH-2 & 55.00 & 93.50 & -41.18 & 0.00 & 70.00 & 87.10 & 103.00 & -15.44 & 0.00 & 87.27 & 124.25 & 88.00 & 41.19 & 0.00 & 60.00 & 88.78 & 55.00 & 61.42 & 0.00 & 0.00 \\
\hline JK-Ishwar & 78.00 & 94.10 & -17.11 & 41.82 & 71.09 & 88.40 & 120.00 & -26.33 & 1.49 & 118.18 & 130.35 & 97.30 & 33.97 & 4.91 & 76.91 & 98.92 & 78.00 & 26.82 & 11.42 & 41.82 \\
\hline JKCH-1947 & 90.60 & 83.40 & 8.63 & 64.73 & 51.64 & 83.30 & 82.05 & 1.52 & -4.36 & 49.18 & 104.40 & 82.48 & 26.58 & -15.98 & 49.96 & 92.77 & 90.60 & 2.40 & 4.49 & 64.73 \\
\hline JKCH-22 & 80.30 & 81.40 & -1.35 & 46.00 & 48.00 & 83.10 & 90.60 & -8.28 & -4.59 & 64.73 & 90.80 & 80.77 & 12.42 & -26.92 & 46.85 & 84.73 & 80.30 & 5.52 & -4.56 & 46.00 \\
\hline Ankur-651 & 90.15 & 90.45 & -0.33 & 63.91 & 64.45 & 87.40 & 118.00 & -25.93 & 0.34 & 114.55 & 81.00 & 95.15 & -14.87 & -34.81 & 73.00 & 86.18 & 90.15 & -4.40 & -2.93 & 63.91 \\
\hline JKCH-1050 & 80.50 & 87.00 & -7.47 & 46.36 & 58.18 & 88.20 & 105.50 & -16.40 & 1.26 & 91.82 & 85.00 & 83.17 & 2.20 & -31.59 & 51.22 & 84.57 & 80.50 & 5.06 & -4.74 & 46.36 \\
\hline PCH-2270 & 69.00 & 85.00 & -18.82 & 25.45 & 54.55 & 40.50 & 115.20 & -64.84 & -53.50 & 109.45 & 87.00 & 85.43 & 1.84 & -29.98 & 55.33 & 65.50 & 69.00 & -5.07 & -26.22 & 25.45 \\
\hline NCEN-3R & 83.60 & 80.00 & 4.50 & 52.00 & 45.45 & 87.90 & 97.00 & -9.38 & 0.92 & 76.36 & 102.90 & 81.53 & 26.21 & -17.18 & 48.24 & 91.47 & 83.60 & 9.41 & 3.03 & 52.00 \\
\hline КDCHН-9810 & 78.00 & 85.50 & -8.77 & 41.82 & 55.45 & 101.55 & 87.25 & 16.39 & 16.59 & 58.64 & 108.10 & 89.82 & 20.35 & -13.00 & 63.31 & 95.88 & 78.00 & 22.92 & 8.00 & 41.82 \\
\hline NCEN-2R & 93.70 & 108.40 & -13.56 & 70.36 & 97.09 & 107.00 & 137.00 & -21.90 & 22.85 & 149.09 & 137.20 & 106.13 & 29.28 & 10.42 & 92.96 & 112.63 & 93.70 & 20.20 & 26.86 & 70.36 \\
\hline JKCH-266 & 75.00 & 97.30 & -22.92 & 36.36 & 76.91 & 102.05 & 81.20 & 25.68 & 17.16 & 47.64 & 74.50 & 89.17 & -16.45 & -40.04 & 62.13 & 83.85 & 75.00 & 11.80 & -5.55 & 36.36 \\
\hline Dhruva & 97.40 & 82.30 & 18.35 & 77.09 & 49.64 & 83.00 & 94.80 & -12.45 & -4.71 & 72.36 & 97.00 & 91.43 & 6.09 & -21.93 & 66.24 & 92.47 & 97.40 & -5.06 & 4.16 & 77.09 \\
\hline K-5316 & 94.40 & 94.10 & 0.32 & 71.64 & 71.09 & 91.00 & 94.50 & -3.70 & 4.48 & 71.82 & 105.70 & 92.38 & 14.42 & -14.93 & 67.96 & 97.03 & 94.40 & 2.79 & 9.29 & 71.64 \\
\hline КDCHН-9632 & 81.50 & 93.30 & -12.65 & 48.18 & 69.64 & 86.90 & 107.00 & -18.79 & -0.23 & 94.55 & 97.50 & 94.40 & 3.28 & -21.53 & 71.64 & 88.63 & 81.50 & 8.75 & -0.17 & 48.18 \\
\hline JK-Varun & 92.45 & 110.00 & -15.95 & 68.09 & 100.00 & 81.80 & 98.00 & -16.53 & -6.08 & 78.18 & 110.10 & 93.20 & 18.13 & -11.39 & 69.45 & 94.78 & 92.45 & 2.52 & 6.76 & 68.09 \\
\hline PCH-2171 & 60.10 & 80.50 & -25.34 & 9.27 & 46.36 & 76.00 & 82.50 & -7.88 & -12.74 & 50.00 & 90.60 & 78.37 & 15.61 & -27.08 & 42.49 & 75.57 & 60.10 & 25.74 & -14.88 & 9.27 \\
\hline JK-Durga & 88.55 & 78.55 & 12.73 & 61.00 & 42.82 & 78.55 & 85.50 & -8.13 & -9.82 & 55.45 & 99.05 & 84.80 & 16.80 & -20.28 & 54.18 & 88.72 & 88.55 & 0.19 & -0.07 & 61.00 \\
\hline JKСН-99 & 90.50 & 92.10 & -1.74 & 64.55 & 67.45 & 84.05 & 121.00 & -30.54 & -3.50 & 120.00 & 100.70 & 100.80 & -0.10 & -18.95 & 83.27 & 91.75 & 90.50 & 1.38 & 3.35 & 64.55 \\
\hline JKCH-1945 & 74.90 & 114.20 & -34.41 & 36.18 & 107.64 & 94.00 & 79.00 & 18.99 & 7.92 & 43.64 & 103.00 & 92.37 & 11.51 & -17.10 & 67.95 & 90.63 & 74.90 & 21.00 & 2.08 & 36.18 \\
\hline JK-Gowri & 88.10 & 75.90 & 16.07 & 60.18 & 38.00 & 78.30 & 98.30 & -20.35 & -10.10 & 78.73 & 108.00 & 81.52 & 32.48 & -13.08 & 48.22 & 91.47 & 88.10 & 3.83 & 3.03 & 60.18 \\
\hline Mean & 83.22 & 90.25 & & & & 84.95 & 101.10 & & & & 102.74 & 90.54 & & & & 90.30 & 83.22 & & & \\
\hline $\operatorname{SEm} \pm$ & \multicolumn{2}{|c|}{6.08} & & & & \multicolumn{2}{|c|}{5.15} & & & & \multicolumn{2}{|c|}{5.09} & & & & \multicolumn{2}{|c|}{2.23} & & & \\
\hline CD at $5 \%$ & \multicolumn{2}{|c|}{17.30} & & & & \multicolumn{2}{|c|}{14.65} & & & & \multicolumn{2}{|c|}{14.49} & & & & \multicolumn{2}{|c|}{6.17} & & & \\
\hline CD at $1 \%$ & \multicolumn{2}{|c|}{23.11} & & & & \multicolumn{2}{|c|}{19.56} & & & & \multicolumn{2}{|c|}{19.34} & & & & \multicolumn{2}{|c|}{8.12} & & & \\
\hline
\end{tabular}


Table.11 Mean performance of Bt and non-Bt hybridsacross three environments for number of monopodials

\begin{tabular}{|c|c|c|c|c|c|c|c|c|c|c|c|c|c|c|c|c|c|c|c|c|}
\hline \multirow[t]{2}{*}{ Genotypes } & \multicolumn{5}{|c|}{$E_{1}$ : Nagpur } & \multicolumn{5}{|c|}{$\mathrm{E}_{2}$ : Dharwad } & \multicolumn{5}{|c|}{$E_{3}$ : Bagalkot } & \multirow{2}{*}{$\begin{array}{c}\text { Mean } \\
\text { Bt }\end{array}$} & \multirow{2}{*}{$\begin{array}{l}\text { Mean } \\
\text { NBt }\end{array}$} & \multicolumn{3}{|c|}{ Across locations } \\
\hline & Bt & NBt & $\begin{array}{c}\% \\
\text { over } \\
\text { NBt }\end{array}$ & $\begin{array}{c}\% \\
\text { over } \\
\text { check }\end{array}$ & $\begin{array}{c}\% \text { over } \\
\text { population } \\
\text { mean }\end{array}$ & Bt & NBt & $\begin{array}{c}\% \\
\text { over } \\
\text { NBt }\end{array}$ & $\begin{array}{c}\% \\
\text { over } \\
\text { check }\end{array}$ & $\begin{array}{c}\% \text { over } \\
\text { population } \\
\text { mean }\end{array}$ & Bt & NBt & $\begin{array}{c}\text { \% over } \\
\text { NBt }\end{array}$ & $\begin{array}{c}\% \\
\text { over } \\
\text { check }\end{array}$ & $\begin{array}{c}\% \text { over } \\
\text { population } \\
\text { mean }\end{array}$ & & & $\begin{array}{c}\% \\
\text { over } \\
\text { NBt }\end{array}$ & $\begin{array}{c}\% \\
\text { over } \\
\text { check }\end{array}$ & $\begin{array}{c}\% \\
\text { over } \\
\text { popu } \\
\text { lation } \\
\text { mean }\end{array}$ \\
\hline JKCH-2245 & 2.40 & 3.00 & -20.00 & 20.00 & 50.00 & 1.80 & 2.12 & -15.09 & -18.18 & -3.64 & 2.50 & 2.70 & -7.41 & -8.09 & -0.74 & 2.23 & 2.61 & -14.56 & -3.46 & 12.99 \\
\hline K-5038 & 2.40 & 2.25 & 6.67 & 20.00 & 12.50 & 2.10 & 1.50 & 40.00 & -4.55 & -31.82 & 2.40 & 2.20 & 9.09 & -11.76 & -19.12 & 2.30 & 1.98 & 16.16 & -0.43 & $\begin{array}{c}- \\
14.29 \\
\end{array}$ \\
\hline $\begin{array}{l}\text { KDCHH- } \\
441\end{array}$ & 3.20 & 3.30 & -3.03 & 60.00 & 65.00 & 3.00 & 2.70 & 11.11 & 36.36 & 22.73 & 3.10 & 2.60 & 19.23 & 13.97 & -4.41 & 3.10 & 2.87 & 8.01 & 34.20 & 24.24 \\
\hline JK-Indra & 2.40 & 2.40 & 0.00 & 20.00 & 20.00 & 2.50 & 2.50 & 0.00 & 13.64 & 13.64 & 2.70 & 2.50 & 8.00 & -0.74 & -8.09 & 2.53 & 2.47 & 2.43 & 9.52 & 6.93 \\
\hline$\overline{\mathrm{RCH}-2}$ & 2.00 & 2.65 & -24.53 & 0.00 & 32.50 & 2.20 & 3.50 & -37.14 & 0.00 & 59.09 & 2.72 & 3.30 & -17.58 & 0.00 & 21.32 & 2.31 & 3.15 & -26.67 & 0.00 & 36.36 \\
\hline JK-Ishwar & 2.90 & 2.40 & 20.83 & 45.00 & 20.00 & 1.80 & 1.60 & 12.50 & -18.18 & -27.27 & 1.90 & 3.40 & -44.12 & -30.15 & 25.00 & 2.20 & 2.47 & -10.93 & -4.76 & 6.93 \\
\hline JKCH-1947 & 2.35 & 2.30 & 2.17 & 17.50 & 15.00 & 1.50 & 1.30 & 15.38 & -31.82 & -40.91 & 0.70 & 1.70 & -58.82 & -74.26 & -37.50 & 1.52 & 1.77 & -14.12 & -34.20 & $\begin{array}{c}- \\
23.38\end{array}$ \\
\hline JKCH-22 & 2.60 & 2.80 & -7.14 & 30.00 & 40.00 & 1.90 & 2.50 & -24.00 & -13.64 & 13.64 & 1.90 & 2.80 & -32.14 & -30.15 & 2.94 & 2.13 & 2.70 & -21.11 & -7.79 & 16.88 \\
\hline Ankur-651 & 2.50 & 2.90 & -13.79 & 25.00 & 45.00 & 2.10 & 2.40 & -12.50 & -4.55 & 9.09 & 2.50 & 2.10 & 19.05 & -8.09 & -22.79 & 2.37 & 2.47 & -4.05 & 2.60 & 6.93 \\
\hline JKCH-1050 & 3.10 & 1.85 & 67.57 & 55.00 & -7.50 & 1.80 & 1.60 & 12.50 & -18.18 & -27.27 & 2.50 & 1.20 & 108.33 & -8.09 & -55.88 & 2.47 & 1.55 & 59.35 & 6.93 & $\begin{array}{c}- \\
32.90 \\
\end{array}$ \\
\hline PCH-2270 & 2.40 & 2.40 & 0.00 & 20.00 & 20.00 & 1.50 & 2.10 & -28.57 & -31.82 & -4.55 & 2.10 & 3.10 & -32.26 & -22.79 & 13.97 & 2.00 & 2.53 & -20.95 & -13.42 & 9.52 \\
\hline $\begin{array}{l}\text { KDCHH- } \\
9810\end{array}$ & 2.60 & 3.10 & -16.13 & 30.00 & 55.00 & 2.00 & 2.20 & -9.09 & -9.09 & 0.00 & 1.40 & 2.90 & -51.72 & -48.53 & 6.62 & 2.00 & 2.73 & -26.74 & -13.42 & 18.18 \\
\hline NCEN-2R & 2.15 & 3.10 & -30.65 & 7.50 & 55.00 & 2.20 & 2.40 & -8.33 & 0.00 & 9.09 & 2.70 & 3.10 & -12.90 & -0.74 & 13.97 & 2.35 & 2.87 & -18.12 & 1.73 & 24.24 \\
\hline JKCH-266 & 2.30 & 1.90 & 21.05 & 15.00 & -5.00 & 1.90 & 2.50 & -24.00 & -13.64 & 13.64 & 1.90 & 1.60 & 18.75 & -30.15 & -41.18 & 2.03 & 2.00 & 1.50 & -12.12 & $\begin{array}{c}- \\
13.42\end{array}$ \\
\hline Dhruva & 2.00 & 2.40 & -16.67 & 0.00 & 20.00 & 1.80 & 2.00 & -10.00 & -18.18 & -9.09 & 2.20 & 3.20 & -31.25 & -19.12 & 17.65 & 2.00 & 2.53 & -20.95 & -13.42 & 9.52 \\
\hline K-5316 & 2.60 & 2.10 & 23.81 & 30.00 & 5.00 & 1.10 & 1.10 & 0.00 & -50.00 & -50.00 & 0.90 & 2.80 & -67.86 & -66.91 & 2.94 & 1.53 & 2.00 & -23.50 & -33.77 & $\begin{array}{c}- \\
13.42\end{array}$ \\
\hline $\begin{array}{l}\text { KDCHH- } \\
9632 \\
\end{array}$ & 2.90 & 3.00 & -3.33 & 45.00 & 50.00 & 2.10 & 2.20 & -4.55 & -4.55 & 0.00 & 3.00 & 3.30 & -9.09 & 10.29 & 21.32 & 2.67 & 2.83 & -5.65 & 15.58 & 22.51 \\
\hline JK-Varun & 2.60 & 2.05 & 26.83 & 30.00 & 2.50 & 2.20 & 2.60 & -15.38 & 0.00 & 18.18 & 0.70 & 1.30 & -46.15 & -74.26 & -52.21 & 1.83 & 1.98 & -7.58 & -20.78 & $\begin{array}{c}- \\
14.29\end{array}$ \\
\hline PCH-2171 & 2.80 & 2.20 & 27.27 & 40.00 & 10.00 & 2.10 & 1.50 & 40.00 & -4.55 & -31.82 & 2.10 & 3.30 & -36.36 & -22.79 & 21.32 & 2.33 & 2.33 & 0.00 & 0.87 & 0.87 \\
\hline JK-Durga & 2.85 & 3.15 & -9.52 & 42.50 & 57.50 & 1.60 & 2.30 & -30.43 & -27.27 & 4.55 & 2.00 & 2.40 & -16.67 & -26.47 & -11.76 & 2.15 & 2.62 & -17.94 & -6.93 & 13.42 \\
\hline JKCH-99 & 2.75 & 3.40 & -19.12 & 37.50 & 70.00 & 2.80 & 3.00 & -6.67 & 27.27 & 36.36 & 3.10 & 3.50 & -11.43 & 13.97 & 28.68 & 2.88 & 3.30 & -12.73 & 24.68 & 42.86 \\
\hline JKCH-1945 & 2.50 & 1.75 & 42.86 & 25.00 & -12.50 & 2.30 & 1.50 & 53.33 & 4.55 & -31.82 & 1.10 & 5.70 & -80.70 & -59.56 & 109.56 & 1.97 & 2.98 & -33.89 & -14.72 & 29.00 \\
\hline JK-Gowri & 2.70 & 3.30 & -18.18 & 35.00 & 65.00 & 2.20 & 2.30 & -4.35 & 0.00 & 4.55 & 2.50 & 2.90 & -13.79 & -8.09 & 6.62 & 2.47 & 2.83 & -12.72 & 6.93 & 22.51 \\
\hline Mean & 2.57 & 2.60 & & & & 2.00 & 2.15 & & & & 2.10 & 2.75 & & & & 2.23 & 2.50 & & & \\
\hline SEm \pm & \multicolumn{2}{|c|}{0.19} & & & & \multicolumn{2}{|c|}{0.25} & & & & \multicolumn{2}{|c|}{0.18} & & & & \multicolumn{2}{|c|}{0.08} & & & \\
\hline
\end{tabular}


Table.12 Mean performance of Bt and non-Bt hybrids across three environments for fuzz percentage

\begin{tabular}{|c|c|c|c|c|c|c|c|c|c|c|c|c|c|c|c|c|c|c|c|c|}
\hline \multirow[t]{2}{*}{ Genotypes } & \multicolumn{5}{|c|}{$E_{1}$ : Nagpur } & \multicolumn{5}{|c|}{$\mathrm{E}_{2}$ : Dharwad } & \multicolumn{5}{|c|}{$\mathrm{E}_{3}$ : Bagalkot } & \multirow{2}{*}{$\begin{array}{c}\text { Mean } \\
\text { Bt }\end{array}$} & \multirow{2}{*}{$\begin{array}{l}\text { Mean } \\
\text { NBt }\end{array}$} & \multicolumn{3}{|c|}{ Across locations } \\
\hline & Bt & NBt & $\begin{array}{c}\text { \% over } \\
\text { NBt }\end{array}$ & $\begin{array}{c}\% \\
\text { over } \\
\text { check }\end{array}$ & $\begin{array}{c}\% \text { over } \\
\text { population } \\
\text { mean }\end{array}$ & Bt & NBt & $\begin{array}{l}\% \\
\text { over } \\
\text { NBt }\end{array}$ & $\begin{array}{c}\% \\
\text { over } \\
\text { check }\end{array}$ & $\begin{array}{c}\% \text { over } \\
\text { population } \\
\text { mean }\end{array}$ & Bt & NBt & $\begin{array}{c}\% \\
\text { over } \\
\text { NBt }\end{array}$ & $\begin{array}{l}\text { \% over } \\
\text { check }\end{array}$ & $\begin{array}{c}\% \text { over } \\
\text { population } \\
\text { mean }\end{array}$ & & & $\begin{array}{c}\% \\
\text { over } \\
\text { NBt }\end{array}$ & $\begin{array}{c}\% \\
\text { over } \\
\text { check }\end{array}$ & $\begin{array}{c}\% \\
\text { over } \\
\text { popu } \\
\text { lation } \\
\text { mean } \\
\end{array}$ \\
\hline JKCH-2245 & 12.45 & 4.90 & 154.08 & -7.09 & -63.43 & 11.00 & 11.95 & -7.95 & -10.93 & -3.24 & 8.95 & 5.15 & 73.79 & -42.99 & -67.20 & 10.80 & 7.33 & 47.34 & 21.85 & -46.96 \\
\hline K-5038 & 7.65 & 11.20 & -31.70 & -42.91 & -16.42 & 11.05 & 10.60 & 4.25 & -10.53 & -14.17 & 12.25 & 15.00 & -18.33 & -21.97 & -4.46 & 10.32 & 12.27 & -15.89 & $\begin{array}{c}- \\
25.33\end{array}$ & -11.22 \\
\hline $\begin{array}{l}\text { KDCHH- } \\
441 \\
\end{array}$ & 3.30 & 7.80 & -57.69 & -75.37 & -41.79 & 12.05 & 10.75 & 12.09 & -2.43 & -12.96 & 13.05 & 14.60 & -10.62 & -16.88 & -7.01 & 9.47 & 11.05 & -14.30 & 31.48 & -20.04 \\
\hline JK-Indra & 2.25 & 2.40 & -6.25 & -83.21 & -82.09 & 8.05 & 8.75 & -8.00 & -34.82 & -29.15 & 15.20 & 11.80 & 28.81 & -3.18 & -24.84 & 8.50 & 7.65 & 11.11 & $\begin{array}{c}- \\
38.49\end{array}$ & -44.65 \\
\hline $\mathrm{RCH}-2$ & 13.40 & 9.50 & 41.05 & 0.00 & -29.10 & 12.35 & 10.90 & 13.30 & 0.00 & -11.74 & 15.70 & 14.50 & 8.28 & 0.00 & -7.64 & 13.82 & 11.63 & 18.83 & 0.00 & -15.85 \\
\hline JK-Ishwar & 29.80 & 1.45 & 1955.17 & 122.39 & -89.18 & 11.95 & 10.00 & 19.50 & -3.24 & -19.03 & 10.20 & 12.05 & -15.35 & -35.03 & -23.25 & 17.32 & 7.83 & 121.20 & 25.33 & -43.34 \\
\hline JKCH-1947 & 9.30 & 9.40 & -1.06 & -30.60 & -29.85 & 12.10 & 9.45 & 28.04 & -2.02 & -23.48 & 11.95 & 11.25 & 6.22 & -23.89 & -28.34 & 11.12 & 10.03 & 10.87 & 19.54 & -27.42 \\
\hline JKCH-22 & 9.75 & 12.75 & -23.53 & -27.24 & -4.85 & 12.50 & 12.50 & 0.00 & 1.21 & 1.21 & 11.75 & 14.20 & -17.25 & -25.16 & -9.55 & 11.33 & 13.15 & -13.84 & $\begin{array}{c}- \\
18.02\end{array}$ & -4.85 \\
\hline Ankur-651 & 4.30 & 5.90 & -27.12 & -67.91 & -55.97 & 13.90 & 8.30 & 67.47 & 12.55 & -32.79 & 12.00 & 9.85 & 21.83 & -23.57 & -37.26 & 10.07 & 8.02 & 25.56 & $\begin{array}{c}- \\
27.13\end{array}$ & -41.97 \\
\hline JKCH-1050 & 3.70 & 17.40 & -78.74 & -72.39 & 29.85 & 9.10 & 9.40 & -3.19 & -26.32 & -23.89 & 11.20 & 10.25 & 9.27 & -28.66 & -34.71 & 8.00 & 12.35 & -35.22 & 42.11 & -10.64 \\
\hline$\overline{\text { PCH-2270 }}$ & 3.50 & 12.80 & -72.66 & -73.88 & -4.48 & 10.40 & 8.90 & 16.85 & -15.79 & -27.94 & 14.55 & 13.40 & 8.58 & -7.32 & -14.65 & 9.48 & 11.70 & -18.97 & $\begin{array}{c}- \\
31.40\end{array}$ & -15.34 \\
\hline NCEN-3R & 12.95 & 11.40 & 13.60 & -3.36 & -14.93 & 9.30 & 7.60 & 22.37 & -24.70 & -38.46 & 10.55 & 12.50 & -15.60 & -32.80 & -20.38 & 10.93 & 10.50 & 4.10 & 20.91 & -24.02 \\
\hline $\begin{array}{l}\text { KDCHH- } \\
9810 \\
\end{array}$ & 8.20 & 12.10 & -32.23 & -38.81 & -9.70 & 10.75 & 11.70 & -8.12 & -12.96 & -5.26 & 11.90 & 14.00 & -15.00 & -24.20 & -10.83 & 10.28 & 12.60 & -18.41 & $\begin{array}{c}- \\
25.62\end{array}$ & -8.83 \\
\hline NCEN-2R & 7.40 & 5.50 & 34.55 & -44.78 & -58.96 & 14.10 & 9.85 & 43.15 & 14.17 & -20.24 & 11.00 & 12.95 & -15.06 & -29.94 & -17.52 & 10.83 & 9.43 & 14.85 & $\begin{array}{c}- \\
21.64\end{array}$ & -31.77 \\
\hline JKCH-266 & 10.85 & 7.25 & 49.66 & -19.03 & -45.90 & 9.05 & 9.80 & -7.65 & -26.72 & -20.65 & 12.50 & 14.55 & -14.09 & -20.38 & -7.32 & 10.80 & 10.53 & 2.56 & $\begin{array}{c}- \\
21.85\end{array}$ & -23.81 \\
\hline Dhruva & 5.55 & 1.80 & 208.33 & -58.58 & -86.57 & 12.05 & 10.30 & 16.99 & -2.43 & -16.60 & 10.10 & 10.60 & -4.72 & -35.67 & -32.48 & 9.23 & 7.57 & 21.93 & 33.21 & -45.22 \\
\hline K-5316 & 5.20 & 3.55 & 46.48 & -61.19 & -73.51 & 10.60 & 9.00 & 17.78 & -14.17 & -27.13 & 11.05 & 12.75 & -13.33 & -29.62 & -18.79 & 8.95 & 8.43 & 6.17 & $\begin{array}{c}- \\
35.24\end{array}$ & -39.00 \\
\hline $\begin{array}{l}\text { KDCHH- } \\
9632 \\
\end{array}$ & 20.65 & 2.35 & 778.72 & 54.10 & -82.46 & 10.45 & 9.20 & 13.59 & -15.38 & -25.51 & 10.30 & 10.55 & -2.37 & -34.39 & -32.80 & 13.80 & 7.37 & 87.25 & -0.14 & -46.67 \\
\hline JK-Varun & 17.95 & 6.70 & 167.91 & 33.96 & -50.00 & 13.45 & 16.55 & -18.73 & 8.91 & 34.01 & 14.70 & 14.85 & -1.01 & -6.37 & -5.41 & 15.39 & 12.70 & 21.18 & 11.36 & -8.10 \\
\hline
\end{tabular}


Int.J.Curr.Microbiol.App.Sci (2018) 7(2): 3384-3409

\begin{tabular}{|c|c|c|c|c|c|c|c|c|c|c|c|c|c|c|c|c|c|c|c|c|}
\hline PCH-2171 & 18.30 & 1.50 & 1120.00 & 36.57 & -88.81 & 12.70 & 11.65 & 9.01 & 2.83 & -5.67 & 12.65 & 14.65 & -13.65 & -19.43 & -6.69 & 14.55 & 9.27 & 56.96 & 5.28 & -32.92 \\
\hline JK-Durga & 12.45 & 10.10 & 23.27 & -7.09 & -24.63 & 8.85 & 10.95 & -19.18 & -28.34 & -11.34 & 14.20 & 10.00 & 42.00 & -9.55 & -36.31 & 11.83 & 10.35 & 14.30 & $\begin{array}{c}- \\
14.40\end{array}$ & -25.11 \\
\hline JKCH-99 & 1.40 & 11.10 & -87.39 & -89.55 & -17.16 & 11.80 & 10.25 & 15.12 & -4.45 & -17.00 & 10.50 & 10.70 & -1.87 & -33.12 & -31.85 & 7.90 & 10.68 & -26.03 & 42.84 & -22.72 \\
\hline JKCH-1945 & 6.10 & 13.10 & -53.44 & -54.48 & -2.24 & 7.80 & 10.10 & -22.77 & -36.84 & -18.22 & 12.75 & 9.95 & 28.14 & -18.79 & -36.62 & 8.88 & 11.05 & -19.64 & $\begin{array}{l}- \\
35.75\end{array}$ & -20.04 \\
\hline JK-Gowri & 12.40 & 6.05 & 104.96 & -7.46 & -54.85 & 13.60 & 12.70 & 7.09 & 10.12 & 2.83 & 14.40 & 9.50 & 51.58 & -8.28 & -39.49 & 13.47 & 9.42 & 42.99 & -2.53 & -31.84 \\
\hline Mean & 9.95 & 7.83 & 176.08 & -25.75 & -41.57 & 11.21 & 10.46 & 8.79 & -9.23 & -15.30 & 12.23 & 12.07 & 5.01 & -22.10 & -23.12 & 11.13 & 10.12 & 14.37 & $\begin{array}{c}- \\
19.46\end{array}$ & -26.77 \\
\hline SEm \pm & \multicolumn{2}{|c|}{0.70} & & & & \multicolumn{2}{|c|}{0.94} & & & & \multicolumn{2}{|c|}{1.32} & & & & \multicolumn{2}{|c|}{0.41} & & & \\
\hline CD at $5 \%$ & \multicolumn{2}{|c|}{1.98} & & & & \multicolumn{2}{|c|}{2.67} & & & & \multicolumn{2}{|c|}{3.77} & & & & \multicolumn{2}{|c|}{1.15} & & & \\
\hline CD at $1 \%$ & \multicolumn{2}{|c|}{2.66} & & & & \multicolumn{2}{|c|}{3.56} & & & & \multicolumn{2}{|c|}{5.04} & & & & \multicolumn{2}{|c|}{1.51} & & & \\
\hline
\end{tabular}

Table.14 Analysis of variance in respect of yield and oil related traits in Bt and non-Bt cotton hybrids across three environments

\begin{tabular}{|c|c|c|c|c|c|c|c|c|c|c|c|c|c|}
\hline $\begin{array}{l}\text { Source of } \\
\text { variation }\end{array}$ & d.f. & $\begin{array}{l}\text { Seed cotton } \\
\text { Yield (kg/ha) }\end{array}$ & $\begin{array}{c}\text { Plant } \\
\text { height } \\
(\mathrm{cm})\end{array}$ & $\begin{array}{c}\text { Monopodial } \\
\text { branches }\end{array}$ & $\begin{array}{c}\text { Sympodial } \\
\text { branches }\end{array}$ & $\begin{array}{c}\text { Bolls per } \\
\text { plant }\end{array}$ & $\begin{array}{c}\text { 20-bolls } \\
\text { weight (g) }\end{array}$ & $\begin{array}{c}\text { GOT } \\
(\%)\end{array}$ & $\begin{array}{l}\text { Seed } \\
\text { index }\end{array}$ & Oil (\%) & $\begin{array}{c}\text { Fuzz } \\
(\%)\end{array}$ & Hull (\%) & $\begin{array}{c}\text { Kernel } \\
(\%)\end{array}$ \\
\hline Genotypes & 47 & $320147.34 * *$ & 187.09 & $0.538^{*}$ & 2.08 & 25.42 & $146.87 * *$ & $15.80 * *$ & $2.36^{* *}$ & $906 * *$ & 14.63 & 21.23 & 21.22 \\
\hline $\begin{array}{l}\text { Environment } \\
(e-1)\end{array}$ & 2 & $13166.84 * *$ & $4976.47 * *$ & 3.25 & $32.81 * *$ & $905.11 * *$ & $12663.70 * *$ & $66.13 * *$ & $39.01 * *$ & $55.59 * *$ & $128.49 * *$ & $690.82 * *$ & $687.38 * *$ \\
\hline $\begin{array}{l}\text { Genotypes } \times \\
\text { environment }\end{array}$ & 94 & 167981.91 & $146.29 * *$ & $0.32 *$ & 3.09 & $23.90 * *$ & 85.88 & 4.05 & $0.70^{*}$ & $2.25 * *$ & $13.01 * *$ & 24.07 & 24.06 \\
\hline $\begin{array}{l}\text { Environment } \\
(\operatorname{var} \times \text { env) }\end{array}$ & 96 & 191913.21 & 246.92 & 0.38 & 3.71 & 42.26 & 347.91 & 5.34 & 1.50 & 3.36 & 15.42 & 37.96 & 37.87 \\
\hline $\begin{array}{l}\text { Environment } \\
\text { (linear) }\end{array}$ & 1 & $2633417.40 * *$ & $9952.34 * *$ & $6.52 * *$ & $65.62 * *$ & $1810.21 * *$ & $25327.55^{* *}$ & $132.10^{* *}$ & $78.03 * *$ & $111.18 * *$ & $256.99 * *$ & $1381.72 * *$ & 1374.52 \\
\hline $\begin{array}{l}\text { Genotype } \times \\
\text { environment } \\
\text { (linear) }\end{array}$ & 47 & $133836.20 * *$ & 179.72 & 0.13 & $4.14 * *$ & $19.98 * *$ & 50.38 & $7.71 * *$ & 0.48 & 2.33 & $20.21 * *$ & $30.21 * *$ & $30.20 * *$ \\
\hline $\begin{array}{l}\text { Pooled } \\
\text { deviation }\end{array}$ & 48 & 197914.88 & $110.53 * *$ & $0.49 * *$ & $2.00 * *$ & $17.45^{* *}$ & $118.84 * *$ & 0.38 & $0.90 * *$ & $2.12 * *$ & $5.70 * *$ & $17.55 * *$ & $17.54 * *$ \\
\hline Pooled error & 141 & 39718.07 & 59.71 & 0.087 & 2.35 & 9.84 & 74.01 & 3.59 & 0.167 & 1.08 & 2.09 & 20.65 & 20.43 \\
\hline
\end{tabular}

*, **: Significant at $5 \%$ and $1 \%$ level of probability 
Table.13 Analysis of variance for different characters in Bt and non-Bt hybrids for stability across three environments

\begin{tabular}{|c|c|c|c|c|}
\hline \multirow{2}{*}{$\begin{array}{l}\text { SI. } \\
\text { No. }\end{array}$} & \multirow[t]{2}{*}{ Character } & \multicolumn{3}{|c|}{ Mean squares due to genotype } \\
\hline & & $\mathbf{E}_{1}$ & $\mathbf{E}_{2}$ & $\mathbf{E}_{3}$ \\
\hline 1. & Seed cotton yield & $642891.23 * *$ & $173553.02 * *$ & $495774.98 * *$ \\
\hline 2. & Plant height & $263.74 * *$ & $264.84 * *$ & $430.77 * *$ \\
\hline 3. & Monopodial branches & $0.33 * *$ & $0.49 * *$ & $1.53 \% *$ \\
\hline 4. & Sympodial branches & $4.52 * *$ & $2.67 * *$ & $9.36 * * *$ \\
\hline 5. & Bolls per plant & 68.66 & $24.35^{* *}$ & $53.44 * *$ \\
\hline 6. & 20-bolls weight & $360.62 * *$ & $161.20 * *$ & $115.45 * *$ \\
\hline 7. & Ginning outturn & $22.01^{* *}$ & $10.64^{*}$ & $15.17 * *$ \\
\hline 8. & Seed index & $3.80 * *$ & $1.92 * *$ & $1.83 * *$ \\
\hline 9. & Oil content & $10.65^{* *}$ & $2.09 * *$ & $14.39 \% *$ \\
\hline 10. & Fuzz percentage & $65.82^{* *}$ & $6.71 * *$ & $8.82^{* * * *}$ \\
\hline 11. & Hull content & $72.65^{* *}$ & $20.42 * *$ & $45.68 * *$ \\
\hline 12. & Kernel content & $72.62 * *$ & 20.37 & $45.68 * *$ \\
\hline
\end{tabular}

$*, * *$ : Significant at $5 \%$ and $1 \%$ level of probability

Table.15 Estimates of stability parameters

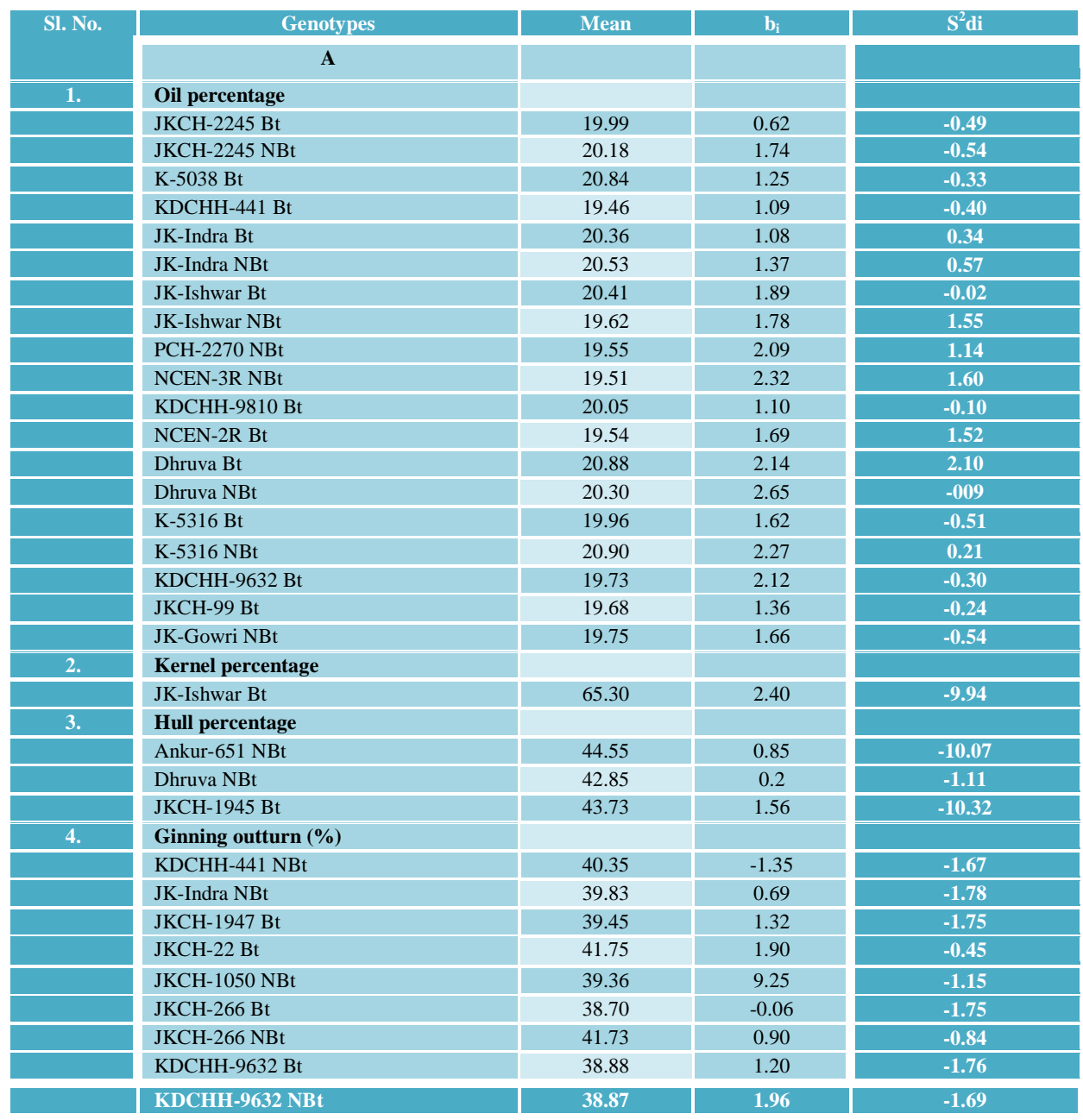


Contd....

\begin{tabular}{|c|c|c|c|c|}
\hline Sl. No. & Genotypes & Mean & $\mathbf{b}_{\mathrm{i}}$ & $S^{2} d i$ \\
\hline \multirow[t]{12}{*}{5.} & Seed index & & & \\
\hline & $\mathrm{JKCH}-2245 \mathrm{NBt}$ & 8.80 & 0.43 & 0.03 \\
\hline & $\mathrm{K}-5038 \mathrm{Bt}$ & 8.60 & 0.58 & -0.03 \\
\hline & KDCHH-441 NBt & 8.65 & 0.69 & 0.23 \\
\hline & $\mathrm{RCH}-2 \mathrm{NBt}$ & 8.32 & 1.97 & -0.07 \\
\hline & $\mathrm{PCH}-2270 \mathrm{NBt}$ & 8.22 & 1.42 & -0.08 \\
\hline & NCEN-3R NBt & 9.07 & 0.84 & -0.07 \\
\hline & KDCHH-9810 Bt & 8.40 & 1.15 & 0.11 \\
\hline & Dhruva NBt & 8.88 & 1.30 & 0.00 \\
\hline & KDCHH-9632 Bt & 8.68 & 1.24 & -0.01 \\
\hline & JK-Durga Bt & 7.00 & 1.31 & -0.07 \\
\hline & JK-Durga NBt & 7.82 & 1.28 & $0.80 * *$ \\
\hline \multirow[t]{7}{*}{$\overline{6 .}$} & $\begin{array}{l}\text { Seed cotton yield } \\
\text { (kg/ha) }\end{array}$ & & & \\
\hline & JKCH-2245 NBt & 1665.33 & 0.38 & 17412.80 \\
\hline & $\mathrm{K}-5038 \mathrm{Bt}$ & 1820.33 & 1.02 & 43404.80 \\
\hline & KDCHH-441 Bt & 1651.00 & 0.34 & -17053.10 \\
\hline & NCEN-2R NBt & 1763.33 & 0.08 & 34261.10 \\
\hline & Dhruva Bt & 1643.00 & 3.14 & 1614.80 \\
\hline & JKCH-99 Bt & 1577.33 & 0.26 & 2472.40 \\
\hline \multirow[t]{6}{*}{7.} & Number of sympodia & & & \\
\hline & PCH-2270 NBt & 15.37 & 4.53 & 0.32 \\
\hline & KDCHH-9810 Bt & 15.43 & 1.98 & -1.14 \\
\hline & KDCHH-9632 NBt & 15.67 & 6.04 & 0.42 \\
\hline & $\mathrm{JK}-$ Varun Bt & 19.73 & -1.43 & 0.16 \\
\hline & JKCH-99 Bt & 16.20 & 2.50 & -1.18 \\
\hline \multirow[t]{5}{*}{8.} & Bolls per plant & & & \\
\hline & $\mathrm{KDCHH}-441 \mathrm{NBt}$ & 32.55 & 0.84 & 7.85 \\
\hline & NCEN-3R NBt & 30.00 & 0.48 & -2.28 \\
\hline & KDCHH-9632 Bt & 32.83 & 1.71 & 66.42 \\
\hline & JKCH-99 Bt & 34.67 & 1.22 & 4.83 \\
\hline \multirow[t]{4}{*}{9.} & Twenty boll weight & & & \\
\hline & JK-Indra Bt & 87.33 & 0.70 & -33.23 \\
\hline & JK-Ishwar Bt & 90.50 & 1.03 & -25.78 \\
\hline & KDCHH-9810 Bt & 91.17 & 1.06 & -32.57 \\
\hline \multirow[t]{8}{*}{10.} & Plant height (cm) & & & \\
\hline & $\mathrm{JKCH}-2245 \mathrm{Bt}$ & 98.43 & 0.49 & -27.90 \\
\hline & KDCHH-441 NBt & 97.17 & 1.14 & -9.80 \\
\hline & JK-Ishwar Bt & 97.30 & 2.08 & -20.70 \\
\hline & JK-Ishwar NBt & 98.92 & 2.71 & -13.40 \\
\hline & NCEN-2R NBt & 112.63 & 2.19 & -29.70 \\
\hline & $\mathrm{K}-5316 \mathrm{NBt}$ & 97.03 & 0.66 & -1.10 \\
\hline & JKCH-99 Bt & 100.80 & 1.68 & -2.40 \\
\hline
\end{tabular}


Contd....

\begin{tabular}{|c|c|c|c|c|}
\hline Sl. No. & Genotypes & Mean & $\mathbf{b}_{\mathrm{i}}$ & $\mathbf{S}^{2} \mathrm{di}$ \\
\hline \multirow[t]{8}{*}{11.} & Number of monopodia & & & \\
\hline & KDCHH-9810 NBt & 2.73 & 1.80 & -0.04 \\
\hline & NCEN-2R NBt & 2.87 & 1.47 & -0.01 \\
\hline & KDCHH-9632 Bt & 2.67 & 1.73 & 0.04 \\
\hline & KDCHH-9632 NBt & 2.83 & 1.82 & 0.15 \\
\hline & JK-Durga NBt & 2.62 & 1.45 & 0.10 \\
\hline & JKCH-99 Bt & 2.88 & 0.05 & 0.03 \\
\hline & JKCH-99 NBt & 3.30 & 0.89 & -0.01 \\
\hline \multirow[t]{10}{*}{12.} & Fuzz percentage & & & \\
\hline & $\mathrm{RCH}-2 \mathrm{Bt}$ & 13.82 & 0.62 & 2.79 \\
\hline & $\mathrm{JKCH}-22 \mathrm{Bt}$ & 11.33 & 0.66 & -0.22 \\
\hline & JKCH-22 NBt & 13.15 & 0.39 & 0.55 \\
\hline & KDCHH-9810 NBt & 12.60 & 0.53 & 0.49 \\
\hline & JK-Varun Bt & 15.39 & -1.09 & 3.34 \\
\hline & $\mathrm{PCH}-2171 \mathrm{Bt}$ & 14.55 & -1.82 & 2.32 \\
\hline & JK-Durga Bt & 11.83 & 0.37 & 13.12 \\
\hline & JK-Gowri Bt & 13.47 & 0.62 & -1.04 \\
\hline & B & & & \\
\hline \multirow[t]{3}{*}{2.} & Kernel percentage & & & \\
\hline & JK-Indra Bt & 66.60 & 2.95 & $225.47 * * *$ \\
\hline & JK-Durga NBt & 67.62 & -1.29 & $57.68 *$ \\
\hline \multirow[t]{2}{*}{3.} & Hull percentage & & & \\
\hline & NCEN-2R Bt & 48.65 & 0.64 & $55.62 *$ \\
\hline \multirow[t]{3}{*}{4.} & Ginning outturn (\%) & & & \\
\hline & $\mathrm{JK}-\mathrm{V}$ arun $\mathrm{Bt}$ & 42.11 & 1.59 & $-1.73 *$ \\
\hline & JKCH-1945 Bt & 40.00 & 1.52 & $10.24 *$ \\
\hline \multirow[t]{9}{*}{5.} & Seed index & & & \\
\hline & $\mathrm{K}-5038 \mathrm{NBt}$ & 8.85 & 0.56 & $0.78 * * *$ \\
\hline & KDCHH-441 Bt & 8.45 & 1.25 & $0.40 *$ \\
\hline & JK-Ishwar NBt & 8.93 & 1.27 & $0.44 *$ \\
\hline & JKCH-22 NBt & 8.47 & -0.21 & $3.79 * *$ \\
\hline & KDCHH-9810 NBt & 9.20 & 0.48 & $1.74 * *$ \\
\hline & NCEN-2R Bt & 8.22 & 1.87 & $1.47 * *$ \\
\hline & KDCHH-9632 NBt & 8.63 & 1.02 & $0.81 * *$ \\
\hline & JK-Gowri NBt & 8.55 & 0.80 & $0.33 *$ \\
\hline \multirow[t]{8}{*}{6.} & Seed cotton yield $(\mathrm{kg} / \mathrm{ha}$ & & & \\
\hline & $\mathrm{JKCH}-2245 \mathrm{Bt}$ & 2181.67 & -2.18 & $312059.40 *$ \\
\hline & JK-Indra Bt & 2012.00 & -1.62 & $166303.20 * *$ \\
\hline & JK-Indra NBt & 1626.00 & 0.93 & $282994.40 * * 6$ \\
\hline & $\mathrm{JKCH}-22 \mathrm{NBt}$ & 1583.67 & $-0.53 *$ & -19156.50 \\
\hline & NCEN-3R Bt & 2283.33 & -3.56 & $69851.90 * *$ \\
\hline & KDCHH-9810 Bt & 1680.00 & 0.98 & $111126.80 * *$ \\
\hline & NCEN-2R Bt & 1631.00 & 2.70 & $81400.80 * * *$ \\
\hline
\end{tabular}




\begin{tabular}{|c|c|c|c|c|}
\hline Sl. No. & Genotypes & Mean & $\mathbf{b}_{\mathrm{i}}$ & $S^{2}$ di \\
\hline \multirow[t]{2}{*}{7.} & Dhruva NBt & 1831.00 & 0.25 & $140947.00 * *$ \\
\hline & JKCH-1945 NBt & 1762.33 & -1.33 & $517486.20 * *$ \\
\hline \multirow[t]{5}{*}{8.} & Bolls per plant & & & \\
\hline & NCEN-2R Bt & 31.50 & 0.62 & $25.79 *$ \\
\hline & NCEN-2R NBt & 34.57 & 0.07 & $126.78 * * *$ \\
\hline & $\mathrm{JK}-\mathrm{V}$ arun NBt & 32.23 & 0.91 & $32.9 *$ \\
\hline & JKCH-99 NBt & 30.70 & 0.60 & $23.37 *$ \\
\hline \multirow[t]{6}{*}{9.} & Twenty boll weight & & & \\
\hline & JK-Ishwar NBt & 87.17 & 1.77 & $247.82 *$ \\
\hline & KDCHH-9810 NBt & 92.33 & 1.54 & $650.18 * *$ \\
\hline & Dhruva NBt & 89.90 & 0.99 & $458.75^{* * *}$ \\
\hline & $\mathrm{K}-5316 \mathrm{NBt}$ & 91.67 & 0.62 & $576.49 * *$ \\
\hline & PCH-2171 NBt & 71.83 & 1.21 & $244.56^{* * * *}$ \\
\hline \multirow[t]{3}{*}{10.} & Plant height (cm) & & & \\
\hline & JKCH-2245 NBt & 91.12 & 0.50 & 41.82 \\
\hline & NCEN-2R Bt & 106.13 & 3.02 & $140.90 *$ \\
\hline \multirow[t]{2}{*}{11.} & Number of monopodia & & & \\
\hline & JKCH-1945 NBt & 2.98 & 2.33 & $10.32 * *$ \\
\hline \multirow[t]{2}{*}{12.} & Fuzz percentage & & & \\
\hline & $\mathrm{K}-5038 \mathrm{NBt}$ & 12.27 & 1.06 & $4.3 *$ \\
\hline \multirow[t]{11}{*}{ Iiiii;'/ } & JK-Ishwar Bt & 17.32 & $-6.26^{*}$ & $24.76 * *$ \\
\hline & JKCH-1050 NBt & 12.35 & -2.34 & $8.33 *$ \\
\hline & KDCHH-9632 Bt & 13.80 & $-3.33^{*}$ & $9.92 * *$ \\
\hline & $\mathrm{JK}-$ Varun NBt & 12.70 & 2.69 & $15.63 * *$ \\
\hline & $\mathbf{C}$ & & & \\
\hline & Seed cotton yield $(\mathrm{kg} / \mathrm{ha}$ & & & \\
\hline & JKCH-22 NBt & 1583.67 & $-0.53^{*}$ & -19156.50 \\
\hline & D & & & \\
\hline & Fuzz percentage & & & \\
\hline & JK-Ishwar Bt & 17.32 & $-6.26^{*}$ & $24.76 * *$ \\
\hline & KDCHH-9632 Bt & 13.80 & $-3.33 *$ & $9.92 * * *$ \\
\hline
\end{tabular}

Contd....

Note:

$\mathrm{A}=$ High mean; bi $\approx 1 ; \mathrm{S}^{2}$ di $-\mathrm{NS} ;$ Remarks - Well adapted to all three environments

$\mathrm{B}=$ High mean; bi $\approx \mathrm{NS} ; \mathrm{S}^{2} \mathrm{di}-\mathrm{S} ;$ Remarks - Not well adapted to all three environments

$\mathrm{C}=$ High mean; $\mathrm{bi} \approx \mathrm{S} ; \mathrm{S}^{2} \mathrm{di}-\mathrm{NS} ;$ Remarks - Not well adapted to all three environments

$\mathrm{D}=$ High mean; bi $\approx \mathrm{S} ; \mathrm{S}^{2} \mathrm{di}-\mathrm{S} ;$ Remarks - Not well adapted to all three environments

"pkj,./uikLOP:"

Table.16 Genotypes with differential adaptability to the three environments for seed cotton yield

\begin{tabular}{|l|}
\hline \multicolumn{1}{|c|}{ Genotypes } \\
\hline A \\
\hline JKCH-2245 NBt \\
\hline K-5038 Bt \\
\hline KDCHH-441 Bt \\
\hline NCEN-2R NBt \\
\hline Dhruva Bt \\
\hline
\end{tabular}

\begin{tabular}{c|c}
\hline $\mathbf{E}_{1}$ (Nagpur) & $\mathbf{E}_{2}$ (Dharwad) \\
\hline$\sqrt{ }$ & $\sqrt{ }$ \\
$\sqrt{ }$ & $\sqrt{ }$ \\
$\sqrt{ }$ & $\sqrt{ }$ \\
$\sqrt{ }$ & $\sqrt{ }$ \\
$\sqrt{ }$ & $\sqrt{ }$ \\
\hline
\end{tabular}

$E_{3}$ (Bagalkot)

JKCH-99 Bt

$\sqrt{ }$

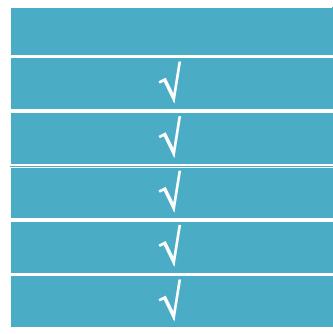




\section{List of location specific hybrids}

\begin{tabular}{|l|}
\hline B \\
\hline JK-Indra Bt \\
\hline JK-Indra NBt \\
\hline NCEN-3R Bt \\
\hline KDCHH-9810 Bt \\
\hline NCEN-2R Bt \\
\hline Dhruva NBt \\
\hline JKCH-1945 Bt \\
\hline C \\
\hline JKCH-22 NBt \\
\hline
\end{tabular}

\begin{tabular}{|c|c|}
\hline $\mathbf{E}_{1}$ (Nagpur) & $\mathbf{E}_{2}$ (Dharwad) \\
\hline$\sqrt{ }$ & \\
$\sqrt{ }$ & \\
$\sqrt{ }$ & \\
$\sqrt{ }$ & $\sqrt{ }$ \\
$\sqrt{ }$ & $\sqrt{ }$ \\
$\sqrt{ }$ & \\
$\sqrt{ }$ & \\
\end{tabular}

\section{$\mathbf{E}_{3}$ (Bagalkot)}

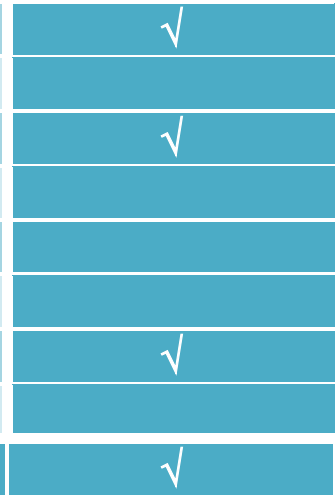

Note:

$\mathrm{A}=$ High mean; bi $\approx 1 ; \mathrm{S}^{2}$ di $-\mathrm{NS} ;$ Remarks - Well adapted to all three environments

$\mathrm{B}=$ High mean; bi - NS; $\mathrm{S}^{2}$ di $-\mathrm{S} ;$ Remarks - Not well adapted to all three environments

$\mathrm{C}=$ High mean; bi $-\mathrm{S} ; \mathrm{S}^{2} \mathrm{di}-\mathrm{NS}$; Remarks - Not well adapted to all three environments

$\sqrt{ }=$ Adaptability

\section{Genotype-environment interaction}

\begin{tabular}{|c|c|c|c|c|}
\hline Source of variation & D.f & SS & MS & F value \\
\hline Genotype (G) & $(g-1)$ & $\Sigma_{\mathrm{i}} \Sigma_{\mathrm{j}} \mathrm{Y}_{\mathrm{ij}}^{2}-\mathrm{CF}$ & $\mathrm{MS}_{1}$ & $\mathrm{MS}_{1} / \mathrm{MS}_{3}$ \\
\hline Environment (E) & $(\mathrm{n}-1)$ & $1 / \mathrm{n} \Sigma_{\mathrm{j}} \mathrm{Y}_{\mathrm{i}}^{2}-1 / \mathrm{n} \Sigma_{\mathrm{j}} \mathrm{Y}_{\mathrm{i}}^{2}$ & & \\
\hline $\mathrm{G} \times \mathrm{E}$ & $(\mathrm{g}-1)(\mathrm{n}-1)$ & $\Sigma_{\mathrm{i}} \Sigma_{\mathrm{j}} \mathrm{Y}_{\mathrm{ij}}^{2}-1 / \mathrm{n} \Sigma_{\mathrm{j}} \mathrm{Y}_{\mathrm{i}}^{2}$ & & \\
\hline Environment (linear) & 1 & $1 / \mathrm{g}\left[(\Sigma \mathrm{j} Y \mathrm{j}-\mathrm{Ij})^{2} \Sigma_{\mathrm{j}} \mathrm{Ij}^{2}\right]$ & & \\
\hline $\begin{array}{l}\text { Genotype } \times \text { Environme } \\
\text { nt (Linear) }\end{array}$ & $(\mathrm{g}-1)$ & 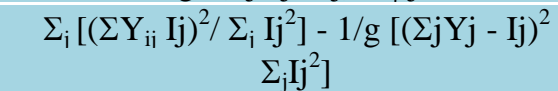 & $\mathrm{MS}_{2}$ & $\mathrm{MS}_{2} / \mathrm{MS}_{3}$ \\
\hline Pooled deviation & $g(n-2)$ & $\Sigma_{\mathrm{i}} \Sigma_{\mathrm{i}} \mathrm{d}^{2} \mathrm{ij}{ }^{2}$ & $\mathrm{MS}_{3}$ & $\mathrm{MS}_{3} / \mathrm{MS}_{4}$ \\
\hline Genotype 1 & $(n-2)$ & {$\left[\Sigma_{\mathrm{i}} \mathrm{Yij}^{2}-\mathrm{Yi}^{2} / \mathrm{n}\right]-\left[\Sigma_{\mathrm{i}} \mathrm{Yij} \mathrm{Ij}^{2} / \Sigma_{\mathrm{i}} \mathrm{Ij}^{2}\right.$} & & \\
\hline Genotype G & $(n-2)$ & {$\left[\Sigma_{\mathrm{i}} \mathrm{Yg}_{\mathrm{i}}{ }^{2}-\mathrm{Yg} .{ }^{2} / \mathrm{n}\right]-\left[\Sigma_{\mathrm{i}} \mathrm{Ygj} \mathrm{Ij}\right]^{2} / \Sigma_{\mathrm{i}} \mathrm{Ij}^{2}$} & & \\
\hline Pooled error & $\mathrm{n}(\mathrm{g}-1)(\mathrm{r}-1)$ & & $\mathrm{MS}_{4}$ & \\
\hline Total & $(n g-1)$ & $\Sigma_{\mathrm{i}} \Sigma_{\mathrm{j}} \mathrm{Yi}_{\mathrm{j}}^{2}-\mathrm{CF}$ & TSS & \\
\hline
\end{tabular}

The estimate of deviation from regression

\begin{tabular}{|c|c|c|c|}
\hline Regression & Stability & $\begin{array}{l}\text { Mean } \\
\text { yield }\end{array}$ & Remarks \\
\hline$b_{i}=1$ & Average & High & Well adapted to all the environments \\
\hline$b_{i}=1$ & Average & Low & Poorly adapted to all environments \\
\hline$b_{i}>1$ & $\begin{array}{l}\text { Below } \\
\text { average }\end{array}$ & High & $\begin{array}{l}\text { Specifically adapted to favourable } \\
\text { environments }\end{array}$ \\
\hline $\mathbf{b}_{\mathrm{i}}<1$ & $\begin{array}{c}\text { Above } \\
\text { average }\end{array}$ & High & $\begin{array}{l}\text { Specifically adapted to unfavourable } \\
\text { environments }\end{array}$ \\
\hline
\end{tabular}


Hybrids like JKCH-2245 NBt (20.18\%), K$5038 \mathrm{Bt}(20.84 \%), \mathrm{KDCHH}-441 \mathrm{Bt}(19.46 \%)$, NCEN-2R NBt (19.27\%), Dhruva Bt $(20.88 \%)$ and JKCH-99 Bt (19.68\%) also showed highly stable reaction for oil content across the three locations.

The hybrids viz., JKCH-99 Bt (16.20), KDCHH-441 Bt (14.83), Dhruva Bt (14.43), K-5038 Bt (13.97), NCEN-2R NBt (13.50) and JKCH-2245 NBt (13.08) were significantly superior over experimental mean for sympodial branches per plant. Number of bolls per plant of hybrids viz., JKCH-99 Bt (34.67), NCEN-2R NBt (34.57), KDCHH441 Bt (28.77), JKCH-2245 NBt (23.23), Dhruva Bt (28.17) and K-5038 Bt (26.30) were significantly more than the population mean (27.41) and $\mathrm{RCH}-2 \mathrm{Bt}$ (26.77). For twenty boll weight, NCEN-2R Bt (79.00 g), K-5038 Bt (85.67 g), Dhruva Bt $(89.90 \mathrm{~g})$, KDCHH-441 Bt (81.33 g), JKCH-2245 NBt (73.67 g) and JKCH-99 Bt (79.17 g) showed higher 20 boll weight.

The hybrids viz., JKCH-2245 NBt (36.87\%), K-5038 Bt (37.47), KDCHH-441 Bt (37.70\%), NCEN-2R NBt (36.50\%) and Dhruva Bt (36.60\%) and JKCH-99 Bt $(36.67 \%)$ showed desirable ginning outturn percentage.

With reference to seed index, JKCH-2245 NBt $(8.80 \mathrm{~g}), \mathrm{K}-5038 \mathrm{Bt}(8.60 \mathrm{~g}), \mathrm{KDCHH}-$ $441 \mathrm{Bt}(8.45 \mathrm{~g})$, NCEN-2R NBt $(8.13 \mathrm{~g})$, Dhruva Bt (7.95 g) and JKCH-99 (8.70 g) showed significantly superior performance over population mean $(7.59 \mathrm{~g})$ for seed index.

Some hybrids were found suitable to a specific location. The Bt hybrid JKCH-2245 Bt was found suitable to Nagpur (2700 kg/ha) and Bagalkot $(2230 \mathrm{~kg} / \mathrm{ha})$ as its seed cotton yield was significantly superior over experimental mean (1389.43 and 1260.59 $\mathrm{kg} / \mathrm{ha}$, respectively) in these locations and over $\mathrm{RCH}-2$ Bt (1000 and $800 \mathrm{~kg} / \mathrm{ha})$ at Nagpur and Bagalkot, respectively. JK-Indra (2047 kg/ha) exhibited significant superiority over average mean (1260 kg/ha) as well as RCH-2 Bt (838 kg/ha) at Bagalkot and over mean $(1260 \mathrm{~kg} / \mathrm{ha})$ at Nagpur. The non-Bt hybrids JK-Indra (2050 kg/ha) and KDCHH$9810 \mathrm{Bt}(1950.00 \mathrm{~kg} / \mathrm{ha})$ revealed significant superiority over RCH-2 Bt (1000 kg/ha) as well as experimental mean (1389 kg/ha) and these hybrids were suitable to Nagpur. The deviation from the regression coefficient was significant and regression coefficient was near to 1 for these hybrids.

At Bagalkot location, JKCH-1945 non-Bt revealed better adoption which reflected in its yield $(2327 \mathrm{~kg} / \mathrm{ha})$ being higher than the experimental mean (1260 kg/ha) while Dhruva non-Bt $(2150 \mathrm{~kg} / \mathrm{ha})$ was particularly adapted to Nagpur as its mean seed cotton yield $(2150 \mathrm{~kg} / \mathrm{ha})$ was significantly superior to check $(1000 \mathrm{~kg} / \mathrm{ha})$ and the experimental mean $(1389 \mathrm{~kg} / \mathrm{ha})$.

The NCEN-3R Bt (2125 kg/ha) and KDCHH$9810 \mathrm{Bt}(1950 \mathrm{~kg} / \mathrm{ha})$ hybrids were significantly superior to $\mathrm{RCH}-2 \mathrm{Bt}(1000$ $\mathrm{kg} / \mathrm{ha})$ and the experimental mean (838 $\mathrm{kg} / \mathrm{ha}$ ). The hybrids NCEN-2R Bt (1825 $\mathrm{kg} / \mathrm{ha}$ ) gave significantly higher yield than $\mathrm{RCH}-2$ Bt (1000 kg/ha) as well as the experimental mean (1389 $\mathrm{kg} / \mathrm{ha})$ at Nagpur while, at Dharwad this hybrid (2005 kg/ha) had yield higher than RCH-2 Bt (1685 kg/ha) and the experimental mean $(1589 \mathrm{~kg} / \mathrm{ha})$ proving its adaptability to both places. JKIndra NBt (2050.00 kg/ha) was better adapted to Nagpur as its yield was better than the population mean $(1389 \mathrm{~kg} / \mathrm{ha})$ and the check RCH-2 (1000 kg/ha).

All the above hybrids fitted in the condition of having significantly high mean, regression coefficient not significantly deviating from unity and deviation from regression being 
significant, thus proving their adaptability to a specific location.

Among the hybrids evaluated, JKCH-2245 NBt, K-5038 Bt, KDCHH-441 Bt, NCEN-2R $\mathrm{NBt}$, Dhruva Bt and JKCH-99 Bt hybrids were identified as stable hybrids for seed cotton yield based on stability parameters. These can be cultivated across the cotton cultivation zones. However, there were hybrids very much suitable for a specific location, viz., RCH-2 Bt, NCEN-3R Bt, JKIndra Bt and Dhruva NBt which were suitable for Nagpur (Central zone) location only. JKCH-22 NBt was suitable for Bagalkot while NCEN-2R Bt was suitable for both Nagpur and Dharwad locations.

\section{References}

Anandan, A., Koodalingam, K. and Raveendran, T. S., 2005. Phenotypic stability for yield and its components in hybrids of upland cotton. Agric. Sci. Dig., 25(1) 59-61.

Ansingkar, A. S. and Bhale, N. L., 1984. Screening of ovid: 5ovid. generation of Gossypium arboreum Lioni for stability for yield and other economic characters. Indian J. Agric. Sci., 54(9): 705-711.

Baloch, M. J., Soomro, B. A., Bhutto, H. and Tunio, G. H., 1994. Stability analysis for comparing cotton varieties (Gossypium hirsutum L.). Pakistan J. Scientific and Industrial Res., 37(12): 528-530.

Basu, A. K., 1996. Current genetic research in cotton in India. Genetica, 97(3): 279290.

Bhatade, S. S. and Bhale, N. L., 1983. Studies on stability parameters in Gossypium arboreum. Indian J. Agric. Sci., 53(7): 519-524.

Blanche, S. B., Myers, G. O., Zumba, J. Z., Caldwell, D. and Heyes, J., 2006. Stability comparisons between conventional and near isogenic transgenic cultivar. J. Cotton Sci., 10(1): 17-28.

Dani, R. G., 1999. Genetic improvement of seed oil content, following indirect selection for earliness and fibre yield in cotton (Gossypium hirsutum L.). Adv. Pl. Sci., 12(2): 479-492.

Deshpande, L. A. and Bhale, N. L., 1983. Stability for some new hybrids of upland cotton in the Marathwada region. Indian J. Agric. Sci., 53(7): 524528.

Eberhart, S. A. and Russel, R. A., 1964. Stability parameters for comparing varieties. Crop Sci., 6: 36-40.

El-Dayim, M. A., Naseer, S. A., ElShourbagy, A. F. and El-Mazar, M. F., 1982. Cotton varietal testing in middle and south delta. Egypt. Agric. Res. Rev., 60(9): 47-67.

El-Kadi, D. A., El-Shaarway, S. A., El-Lakny, M. A., El-Moshen, A. A. A. and Attiea, R., 2007. Estimation of stability parameters for some Egyptian cotton cultivars. Egyptian J. Pl. Breeding, 11(3): 345-355.

El-Okkia, A. F. H., El-Hanafy, H. R., Foad, M. H. and El_Kersh, I., 1982. Behaviour of six cultivars of Egyptian cotton (Gossypium barbadense L.) in north delta. Egypt. Agric. Res. Rev., 60(9): 69-90.

Finley and Wilkinson, 1963. The analysis of adaption in a plant breeding programme. Australian J. Agric. Res., 14: 742-754.

Nanjundan, J., Sangwan, R. S., Chhabra, B. S. and Siwach, S. S., 2004. Stability análisis of seed cotton yield and fibre quality characters in desi cotton (Gossypium arboreum L.). J. Cotton Res. Dev., 18(2): 161-163.

Nirania, K. S., Chahabra, B. S. and YagyaDutt, 2004. Genotype $\times$ environment interaction for yield and quality traits in 
GMS based hybrids of G. hirsutum. J. Cotton Res. Dev., 18(2): 128-131.

Opando, R. M. and Ombakho, G. A., 1997. Yield evaluation and stability analysis in newly selected KSA cotton cultivars in western Kenya. African Crop Sci. J., 5(2): 119-125.

Patel, J. C., Mehta, N. P., Patel, U. G. and Maisuria, A. T., 1990. Stability of promising genotypes of desi cotton (Gossypium herbaceum) for boll weight and seed cotton yield. Indian J. Agric. Sci., 60(7): 486-487.

Pund, M. M. and Dev, D. V., 2006. Stability of yield and other quantitative traits in upland cotton. J. Cotton Res. Dev., 20(2): 181-184.

Reddy, Y. R. and Reddy, C. V. C. M., 2007. Stability analysis of tetraploid cotton (Gossypium hirsutum L. and Gossypium barbadense L.). Plant Achieves, 7(2): 733-739.
Saini, D. P., Ranwaha, B. R. and Rokadia, P., 2008. Stability parameters in upland cotton (Gossypium hirsutum L.). J. Cotton Res. Dev., 22(1): 28-30.

Sharma, D. H., Dharminder Pathak, A. K., Atwal and Sangha, M. K., 2009. Genetic variation for some chemical and biochemical characteristics in cotton seed oil. Cotton Res. Dev., 23(1): 1-7.

Vanniarajan, C., 2007. Genotypes grouping based on stability parameters in pigeonpea. 2(2): 120-123.

Xing-Chao Zhu, Yushu Xun, Guo Liping, Ye $\mathrm{Zi}$ Hong, Wang-Hailin, Miao Cheng Duo and Zhao YunLei, 2007. Analysis for genetic effect and heterosis of insect resistant transgenic upland cotton crosses in different ecological environments. Scientia-AgriculturaSinica, 40(5): 1056-1063.

\section{How to cite this article:}

Nagappa, B.H. and Khadi, B.M. 2018. Adaptability of Bt and NBt Cotton Hybrids in Different Locations in Central and Southern India. Int.J.Curr.Microbiol.App.Sci. 7(02): 3384-3409. doi: https://doi.org/10.20546/ijcmas.2018.702.405 